\title{
Parametrization of the average ionization and radiative cooling rates of carbon plasmas in a wide range of density and temperature
}

\author{
J.M. Gil , R. Rodriguez ，R. Florido \\ , J.G. Rubiano , M.A. Mendoza , \\ A. de la Nuez , G. Espinosa , P. Martel , E. Minguez
}

\begin{abstract}
A B S T R A C T
In this work we present an analysis of the influence of the thermodynamic regime on the monochromatic emissivity, the radiative power loss and the radiative cooling rate for optically thin carbon plasmas over a wide range of electron temperature and density assuming steady state situations. Furthermore, we propose analytical expressions depending on the electron density and temperature for the average ionization and cooling rate based on polynomial fittings which are valid for the whole range of plasma conditions considered in this work.
\end{abstract}

\section{Introduction}

A detailed knowledge of the radiation distribution and in particular of the radiative power loss and cooling rate is necessary in order to understand the behavior of plasmas in which radiative heating and cooling processes are important. The radiative power loss is of great interest for thermonuclear fusion device as well as in both laboratory and astrophysical plasmas. For example, it plays an important role in the current decays after disruptions in magnetic nuclear fusion confinement devices in which disruptions are assumed to be caused by strongly radiating impurities and where hot tail runaway or supra-thermal electrons generation is caused by incomplete thermalization of the electron velocity distribution during the rapid plasma cooling [1]. It was recognized that radiation losses from the impurities produced by plasma-wall interaction can hinder the plasma heating to thermonuclear temperatures and the concentration, being 'lethal' for fusion, have been estimated for different impurity species [2,3]. Moreover, while the heat conduction plays a stabilizing role on the thermal stability at the plasma edge, radiation losses from impurities can develop thermal instability $[4,5]$. On the other hand, the impurity radiation at the plasma edge of fusion device can also play a positive role by reducing the heat outflows to the certain wall elements, the anomalous heat and the particle losses from the plasma [6,7] or to mitigate disruption induced problems it has been proposed that 'killer' pellets could be injected into the plasma in order to safely terminate the discharge $[8,9]$. Besides, radiative power loss plays a very important role in the structure and behavior of radiative shock waves present in laboratory plasmas such as the velocity and the stability properties of radiative shock or the characteristics of the radiative precursor [10-13]. And, finally, in many astrophysical systems as radiative blast waves in the context of supernova remnants [14-16], radiative precursor shock waves with applications to the studies of stellar jets [17,18], pulsating stars [19] and 
accretion shock during star formation [20], radiatively collapsing jets relevant for protostellar outflows $[21,22]$ or analysis of X-ray spectra of the cores of clusters of galaxies [23-25].

On the other hand, carbon is one of the most important elements under investigation in the research areas commented above. For example, in astrophysics, due to its abundance in the stars; in magnetic nuclear fusion confinement, because it is likely to be a major plasma-facing wall component in the international experimental reactor (ITER) and it is present as impurity in a lot of devices; and it plays a major role in inertial fusion scenarios, for example, in the direct-drive implosion cores where the deuterium target has a wall of plastic shells. Therefore, calculations of the radiative properties, and in particular the radiative power loss and cooling rate, of carbon plasmas over a wide range of plasma conditions are very useful. The calculations of these radiative properties for optically thin carbon plasma have been carried out by several authors at low densities and over a wide range of temperatures where the coronal equilibrium (CE) is achieved [26-28] and at moderate and high densities where the non-local and local thermodynamic equilibrium (NLTE and LTE) are achieved, respectively $[29,30]$. Furthermore, at low densities, there are some analytical formulas for the cooling rates obtained by means of fittings to a polynomial expressions of the temperature of databases assuming CE [26,31].

In a previous work [32], we presented a systematic calculation of average ionization, ionic and level populations of the optically thin carbon plasma in a wide range of plasma conditions. We showed that the comparison between the average ionization and ion and level populations calculated from collisional-radiative steady state (CRSS), corona and Saha-Boltzmann (SAHA) equations could provide information about the thermodynamic regime of the plasma, i.e. the plasma conditions where CE, LTE and NLTE regimes could be assumed. In the subsequent work [33] we presented a study of spectrally resolved, multigroup and mean opacities of carbon plasmas in a wide range of plasma conditions analyzing the effect of the thermodynamic regimes in these magnitudes. This work is a continuation of these previous ones, where we calculate the spectrally resolved emissivity, the radiative power loss and the radiative cooling rate of optically thin carbon plasmas assuming steady state situations and in a wide range of electron densities and temperatures which cover situations where CE, NLTE and LTE regimes are found. We perform an analysis of the influence of the thermodynamic regime in these quantities and we also present a parametrization of the cooling rate obtained by fitting bi-dimensional polynomial expressions (depending on the electron density and temperature) to our calculations which is valid in a wide range of electron temperatures $(1-1000 \mathrm{eV})$ and electron densities $\left(10^{10}-10^{21} \mathrm{~cm}^{-3}\right)$ covering CE, LTE and NLTE regimes. All the calculations presented in this work were made using ABAKO/RAPCAL computational package [34-36] which is briefly described in the next section. In Section 3 the analysis of the monochromatic emissivity, the radiative power loss and the cooling rate is made and the parametrization of the average ionization and the cooling rate is also presented. Finally, Section 4 is devoted to main conclusions and general remarks.

\section{Theoretical model}

The calculations in this work were performed using the computational package ABAKO/RAPCAL [36] that consists of two codes, ABAKO [35] and RAPCAL [34]. In this section, a brief description of them is made.

\section{1. АВAKO}

In order to determine the plasma level populations, in ABAKO a collisional-radiative steady state (CRSS) model is implemented. Following the standard NLTE modeling approach, where an account of the existing atomic states is made and the microscopic (radiative and collisional) processes connecting these states are identified, a rate equation system describing the population density of the atomic states is built and solved, giving the population distribution. Therefore, to find the level population distribution the following system of rate equations is solved:

$\sum_{\zeta^{\prime} j} N_{\zeta^{\prime} j} \mathbb{R}_{\zeta^{\prime} j \rightarrow \zeta i}^{+}-\sum_{\zeta^{\prime} j} N_{\zeta i} \mathbb{R}_{\zeta_{i, \zeta^{\prime} \zeta^{\prime} j}^{-}}^{-}=0$,

where $N_{\zeta i}$ is the population density of the atomic level $i$ of the ion with charge state $\zeta$. The terms $\mathbb{R}_{\zeta^{\prime} j \rightarrow \zeta i}^{+}$and $\mathbb{R}_{\zeta^{\prime} j \rightarrow \zeta i}$ take into account all the atomic processes which contribute to populate and depopulate the state $\zeta i$, respectively. In this paper no radiation-driven processes are explicitly considered. In ABAKO it is assumed that the system has had enough time to thermalize and, therefore, both the electrons and ions have a Maxwell-Boltzmann type energy distribution. Furthermore, in ABAKO it is also assumed that electron and ion temperatures are equal. Therefore, in the following, it will denote the plasma temperature by the electron temperature $T_{e}$.

The CRSS model implemented in ABAKO is applied for the calculation of the plasma level populations for arbitrary optical depths in both LTE and NLTE conditions using a CRSS model. The CRSS model is solved level by level (or configuration by configuration, depending on the atomic description) and it is applied to low-to-high $Z$ ions under a wide range of plasma conditions: CE, NLTE or LTE, optically thin and thick plasmas. Special care was taken during the development of our CRSS model to achieve an optimal balance between accuracy and computational cost. Hence, analytical expressions have been employed for the rate coefficients of the atomic processes included in the CRSS model, which yield a substantial saving of computational requirements, but still providing satisfactory results in relation to those obtained from more sophisticated codes and experimental data as it has been shown in recent NLTE code comparison workshops [37-39]. The processes included in the CRSS model are the following: collisional ionization [40] and three-body recombination, spontaneous decay, collisional excitation [41] and deexcitation, radiative recombination [42], electron capture and autoionization. We have added between brackets the references wherefrom their approximated analytical 
rate coefficients have been acquired. The rates of the inverse processes are obtained through the detailed balance principle. It is worth pointing out that the autoionizing states are included explicitly. It has been shown that these contributions are critical to the determination of the ionization balance. The cross section of the autoionization is evaluated using detailed balance principle from the electron capture cross section which is obtained from the collisional excitation cross section using the approximation given in Ref. [43].

Since the number of rate equations is large due to the number of atomic levels involved, ABAKO makes use of the technique of sparse matrices to storage the non-zero elements of the coefficient matrix of the system, which implies substantial savings in memory requirement. For the matrix inversion we use iterative procedures [36] because they entail much less memory than direct methods and they are faster as well.

The atomic data employed in this work were obtained from FAC code [44]. The calculations were carried out in the detailed level accounting (DLA) approach. The radiative transition rates in FAC are calculated in the single multipole approximation, and in this work they were obtained in the electric dipole approach. Furthermore, configuration interactions within the levels belonging to the same nonrelativistic configuration have been included since it has been shown that for carbon plasmas, for plasma conditions in which the less ionized ions are present, the effect of configuration interaction is noticeable [45]. In Table 1 the set of relativistic configurations and the numbers of resulting levels and line transitions included for each carbon ion in the calculation of the level populations and opacities is shown. In the table $(n)^{w}$ denotes all the possible relativistic configurations that arise from the shell $n$ with $w$ bound electrons. Finally, the continuum lowering due to the influence of the plasma surrounding is also considered. In this work, this one is calculated by means of the expression due to Stewart and Pyatt [46]. Because of the inclusion of the continuum lowering, the kinetics equations must be solved iteratively, since the atomic data depend on the ionization balance.

\subsection{RAPCAL}

RAPCAL code was developed to obtain several relevant plasma radiative properties such as the monochromatic absorption and emission coefficients, mean and multigroup opacities, source functions, radiative power losses, specific intensities and plasma transmission. A detailed description of the code can be found in [34]. For this work, the interest has been focused on the monochromatic emissivity, the radiative power loss and the radiative cooling rate. The monochromatic emissivity is denoted in this work as $j(v)$ and includes the bound-bound, bound-free and free-free contributions

$j(v)=j_{b b}(v)+j_{b f}(v)+j_{f f}(v)$

where $v$ is the photon frequency. The bound-bound contribution to the emissivity is given by

$j_{b b}(v)=\sum_{\zeta} \sum_{i j} j_{\zeta j \rightarrow \zeta_{i}}(v)$,

with

$j_{\zeta j \rightarrow \zeta_{i}}(v)=\frac{h v}{4 \pi} N_{\zeta j} A_{\zeta j \rightarrow \zeta i} \phi_{i j}(v)$,

where $A_{\zeta j \rightarrow \zeta_{i}}$ is the Einstein coefficient for spontaneous deexcitation [47] between the bound states $j, i$ of the ion $\zeta, h$ is Planck's constant, $N_{\zeta j}$ is the population density of the atomic level $j$ of the ion with charge state $\zeta$ and $\phi_{i j}(v)$ represents the line profile. In its evaluation, natural, Doppler and electron-impact [48] broadenings were included. The line-shape function is applied with the Voigt profile that incorporates all these broadenings. The bound-free contribution to the emissivity is determined by means of

$j_{b f}(v)=\sum_{\zeta, i} \sum_{\zeta, j} j_{\zeta+1 j \rightarrow \zeta i}(v)$,

with

$j_{\zeta+1 j \rightarrow \zeta i}(v)=\frac{h}{2 \pi c^{2}}\left(\frac{1}{2 m_{e}}\right)^{3 / 2} N_{\zeta+1 j} N_{e} f(\varepsilon) \frac{g_{\zeta i}}{g_{\zeta+1 j}}$

$\frac{(h v)^{3}}{\varepsilon^{1 / 2}} \sigma_{\zeta i \rightarrow \zeta+1 j}^{p h o}(v)$,

with $\varepsilon$ being the energy of the free electron, $m_{e}$ the electron mass, $N_{e}$ the electron number density and $g_{\zeta_{i}}$ and $g_{\zeta+1 j}$ are the statistical weights of the $i$ and $j$ levels, respectively. In ABAKO a Maxwell-Boltzmann distribution, $f(\varepsilon)$, at temperature $T_{e}$ for the free electrons is assumed. The photoionization cross section, $\sigma_{\zeta i \rightarrow \zeta+1 j}^{p h o}(v)$, has been evaluated using the semiclassical expression of Kramers [42].

Table 1

Set of configurations and the numbers of relativistic configurations, levels and line transitions considered in the calculations performed by $A B A K O / R A P C A L$ code for carbon ions ( $\left.n \leq 10, n^{\prime} \leq 6\right)$.

\begin{tabular}{|c|c|c|c|c|c|c|}
\hline & C-like & B-like & Be-like & Li-like & He-like & H-like \\
\hline & $(2)^{4}$ & $(2)^{3}$ & $(2)^{2}$ & $(2)^{1}$ & $1 s^{2}$ & $1 s^{1}$ \\
\hline & $(2)^{3} n t$ & $(2)^{2} n l$ & $(2)^{1} n l$ & $1 s^{2} n l$ & $1 s^{1} n l$ & $n l$ \\
\hline & $(2)^{2}(3)^{2}$ & $(2)^{1}(3)^{2}$ & $(3)^{2}$ & $1 s^{1}(2)^{2}$ & $(2)^{2}$ & \\
\hline & $(2)^{2}(3)^{1} n^{\prime} l$ & $(2)^{1}(3)^{1} n^{\prime} l$ & $(3)^{1} n^{\prime} l$ & $1 s^{1}(2)^{1} n^{\prime} l$ & $(2)^{1} n^{\prime} l$ & \\
\hline & & $1 s^{1}(2)^{4}$ & $1 s^{1}(2)^{3}$ & $1 s^{1}(3)^{2}$ & $(3)^{2}$ & \\
\hline & & $1 s^{1}(2)^{3} n^{\prime} l$ & $1 s^{1}(2)^{2} n^{\prime} l$ & $1 s^{1}(3)^{1} n^{\prime} l$ & $(3)^{1} n^{\prime} l$ & \\
\hline Configurations & 1677 & 1299 & 644 & 519 & 352 & 100 \\
\hline Levels & 16,616 & 8871 & 2789 & 2642 & 910 & 100 \\
\hline Transitions & 864,448 & 250,073 & 45,671 & 54,433 & 9386 & 742 \\
\hline
\end{tabular}


For the free-free contributions to the emissivity the Kramers semi-classical expression for the inverse bremsstrahlung cross section has been used [49]

$\sigma_{\zeta}^{i b r}(v)=\frac{16 \pi^{2} e^{2} h^{2} \alpha}{3 \sqrt{3}\left(2 \pi m_{e}\right)^{3 / 2}} \frac{\zeta^{2} N_{e}}{T_{e}^{1 / 2}(h v)^{3}}$.

Assuming a Maxwell-Boltzmann distribution for the free electrons, it is obtained that

$j_{f f}(v)=\frac{32 \pi^{2} e^{4} a_{0}^{2} \alpha^{3}}{\sqrt{3}\left(2 \pi m_{e}\right)^{3 / 2} h}\left(\frac{m_{e}}{2 \pi T_{e}}\right)^{1 / 2} \overline{Z^{2}} N_{i o n} N_{e} e^{-h v / T_{e}}$,

where $N_{\text {ion }}$ is the ion number density. The radiative power loss is evaluated as following [50] in $(\mathrm{eV} / \mathrm{s} / \mathrm{ion})$. For the bound-bound contribution

$P_{b b}\left(N_{e}, T_{e}\right)=\sum_{\zeta} \sum_{i j} h v_{i j} A_{i j} N_{\zeta j}$.

The bound-free contribution is given by

$$
\begin{aligned}
P_{b f}\left(N_{e}, T_{e}\right)= & 4 \pi \sum_{\zeta} \sum_{i j} N_{\zeta i}\left(\frac{N_{\zeta+1 j} N_{\zeta}}{N_{\zeta,} N_{\zeta+1}}\right)^{L T E} \\
& \times \int_{v_{0}}^{\infty} \sigma_{\zeta i \rightarrow \zeta+1 j}^{p h o}(v)\left(\frac{2 h v^{3}}{c^{2}}\right) e^{-h v / T_{e}} d v,
\end{aligned}
$$

where $v_{0}$ is the threshold energy and the LTE population ratio is obtained from the Saha equation. The contribution from the free-free transitions is given for a pure Coulomb field as following [51]

$P_{f f}\left(N_{e}, T_{e}\right)=9.55 \times 10^{-14} N_{e} T_{e}^{1 / 2} \sum_{\zeta} Z_{\zeta}^{2} N_{\zeta}$,

where it has been assumed that the gaunt factor equals to unity. The total radiative power loss is then obtained as the sum of the three contributions, and the cooling rate is evaluated as

$\Lambda\left(N_{e}, T_{e}\right)=\frac{\left(P_{b b}+P_{b f}+P_{f f}\right)}{N_{e} N_{i o n}}$.

Finally, in this work we have performed a polynomial fitting in powers of the logarithm of the electron densities and temperatures of the average ionization and the cooling rates obtained from ABAKO/RAPCAL simulations for the whole range of electron temperatures and densities considered in this work. If $A$ denotes the magnitude to fit, the analytical expression employed for the fitting is the following:

$\log A\left(N_{e}, T_{e}\right)=\sum_{i=0}^{n} \sum_{j=0}^{m} C_{i j}\left(\log N_{e}\right)^{i}\left(\log T_{e}\right)^{j}$.

The maximum degrees of the polynomial fitting were fixed to 7 both for the electron density and electron temperature in order to avoid oscillating behaviors. For the fitting it was required relative errors lower than $1 \%$ for the average ionization and lower than $10 \%$ for the cooling rates. Obviously, as the error imposed becomes more

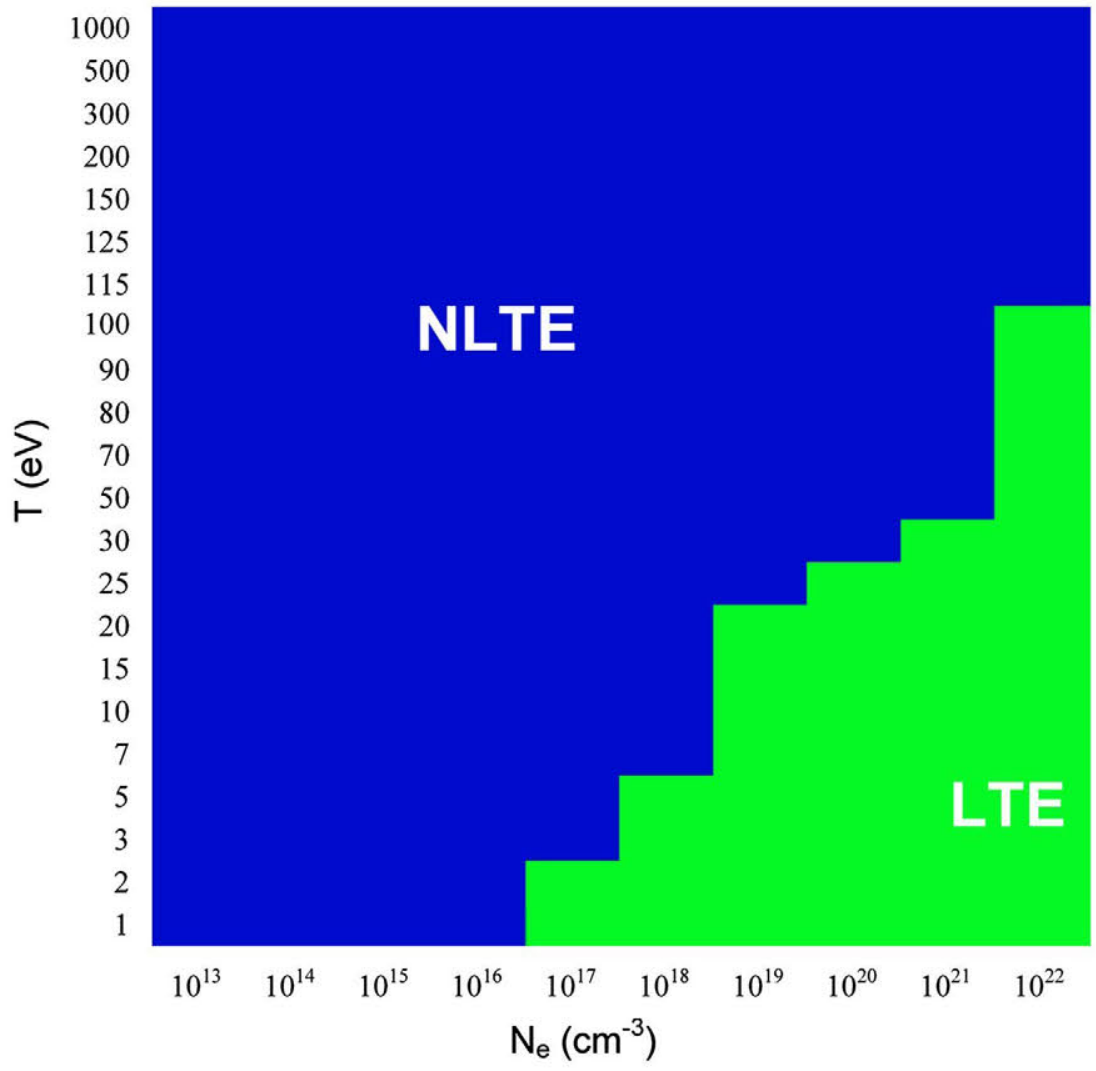

Fig. 1. Thermodynamic regimes for an optically thin carbon plasma in steady state in terms of the electron temperature and density. 
restrictive the number of polynomial functions needed to make the fit of the whole range of plasma conditions increases. We have already used this kind of polynomial fit for $\mathrm{Kr}$ and Xe plasma for ranges of plasma conditions typically found in laboratory experiment of blast waves launched in cluster of gases [52].

\section{Results}

This section is divided into four parts. The first one is devoted to the analysis of the average ionization and the thermodynamic regimes as a function of the plasma conditions. This study will allow us to identify which are the most abundant ions that contribute to the monochromatic emissivity of the optically thin carbon plasmas and also when LTE or NLTE regimes can be assumed. In the second part a study of the influence of the thermodynamic regime into the monochromatic emissivity is carried out, by means of the comparison between the ones calculated using the CRSS and the SAHA equations implemented in ABAKO. In the third part, a similar analysis on the radiative power loss and the radiative cooling rate is performed. Finally, the last part is devoted to the parametrization of the average ionization and radiative cooling rates and the coefficients of the corresponding formulas are listed in Appendix A. The ranges of electron temperatures and densities considered in this work are $1-10^{3} \mathrm{eV}$ and $10^{10}-10^{21} \mathrm{~cm}^{-3}$, respectively.

\subsection{Average ionization and thermodynamic regimes}

The wide range of plasma conditions considered in this work covers different thermodynamic regimes, i.e. LTE or NLTE. The knowledge of the plasma regime for a given density and temperature condition could entail a considerable saving in calculation time since the resolution of SAHA equations is considerably faster than CRSS ones and the complexity is also reduced. Therefore, it is very useful to have criteria that permit us to establish the thermodynamic regime of the plasma for the given electron temperature and density. We have employed a criterion that can state the regime of the whole plasma: when the ion populations, $p_{i}$, calculated from SAHA equations, $p_{i}^{S A H A}$, present a mean deviation $\Delta p$, with respect to those obtained from the CRSS model, $p_{i}^{\text {CRSS }}$, smaller than a certain value imposed $\Delta p^{*}$ then it is considered that LTE regime has been achieved. Otherwise, the plasma is under NLTE conditions. The mean deviation is calculated as

$\Delta p^{L T E}=\sqrt{\frac{\sum_{i}\left(p_{i}^{C R S S}-p_{i}^{S A H A}\right)^{2}}{\sum_{i}\left(p_{i}^{S A H A}\right)^{2}}} \leq \Delta p^{*}$,

where $i$ runs over the whole set of ions included in the calculations. Fixing $\Delta p^{*}=0.1(=10 \%)$ we obtain the map of the thermodynamic regimes showed in Fig. 1. We have checked that, with this value, the criterion proposed to classify the thermodynamic regime of the whole plasma

Table 2

Average ionization calculated using ABAKO for the range of electron densities $10^{10}-10^{15} \mathrm{~g} \mathrm{~cm}^{-3}$ and all the temperatures considered in this work.

\begin{tabular}{|c|c|c|c|c|c|c|}
\hline \multirow[t]{2}{*}{$T_{e}(\mathrm{eV})$} & \multicolumn{6}{|c|}{$N_{e}\left(\mathrm{~cm}^{-3}\right)$} \\
\hline & $10^{10}$ & $10^{11}$ & $10^{12}$ & $10^{13}$ & $10^{14}$ & $10^{15}$ \\
\hline 1.0000 & 0.6700 & 0.6768 & 0.7056 & 0.7747 & 0.8501 & 0.8591 \\
\hline 1.2600 & 0.9409 & 0.9446 & 0.9587 & 0.9762 & 0.9857 & 0.9869 \\
\hline 1.5800 & 0.9834 & 0.9850 & 0.9910 & 0.9970 & 0.9993 & 1.0009 \\
\hline 2.0000 & 1.0049 & 1.0059 & 1.0096 & 1.0166 & 1.0337 & 1.0726 \\
\hline 2.5100 & 1.0990 & 1.1011 & 1.1130 & 1.1563 & 1.3014 & 1.5217 \\
\hline 3.1600 & 1.3998 & 1.4066 & 1.4457 & 1.5452 & 1.7456 & 1.8958 \\
\hline 3.9800 & 1.7481 & 1.7557 & 1.7937 & 1.8549 & 1.9360 & 1.9943 \\
\hline 5.0100 & 1.9549 & 1.9617 & 1.9923 & 2.0239 & 2.0526 & 2.2060 \\
\hline 6.3100 & 2.2851 & 2.3040 & 2.3657 & 2.4030 & 2.4472 & 2.7169 \\
\hline 7.9400 & 2.9939 & 3.0184 & 3.0543 & 3.0686 & 3.0988 & 3.2289 \\
\hline 10.0000 & 3.6843 & 3.6894 & 3.6926 & 3.6944 & 3.7009 & 3.7435 \\
\hline 12.5900 & 3.9246 & 3.9250 & 3.9252 & 3.9254 & 3.9267 & 3.9369 \\
\hline 15.8500 & 3.9794 & 3.9794 & 3.9794 & 3.9795 & 3.9799 & 3.9825 \\
\hline 19.9500 & 3.9931 & 3.9931 & 3.9932 & 3.9932 & 3.9933 & 3.9942 \\
\hline 25.1200 & 3.9973 & 3.9973 & 3.9973 & 3.9973 & 3.9974 & 3.9977 \\
\hline 31.6200 & 3.9994 & 3.9994 & 3.9994 & 3.9994 & 3.9995 & 3.9996 \\
\hline 39.8100 & 4.0101 & 4.0101 & 4.0101 & 4.0101 & 4.0102 & 4.0105 \\
\hline 50.1100 & 4.0993 & 4.0992 & 4.0992 & 4.0992 & 4.0998 & 4.1031 \\
\hline 63.1000 & 4.4416 & 4.4416 & 4.4416 & 4.4418 & 4.4436 & 4.4563 \\
\hline 79.4300 & 4.9202 & 4.9202 & 4.9202 & 4.9204 & 4.9220 & 4.9328 \\
\hline 100.000 & 5.3746 & 5.3746 & 5.3746 & 5.3747 & 5.3753 & 5.3795 \\
\hline 125.890 & 5.7125 & 5.7125 & 5.7125 & 5.7125 & 5.7127 & 5.7140 \\
\hline 158.490 & 5.8856 & 5.8856 & 5.8856 & 5.8856 & 5.8857 & 5.8861 \\
\hline 199.530 & 5.9505 & 5.9505 & 5.9505 & 5.9505 & 5.9506 & 5.9507 \\
\hline 251.190 & 5.9765 & 5.9765 & 5.9765 & 5.9765 & 5.9765 & 5.9766 \\
\hline 316.230 & 5.9877 & 5.9877 & 5.9877 & 5.9877 & 5.9877 & 5.9878 \\
\hline 398.110 & 5.9931 & 5.9931 & 5.9931 & 5.9931 & 5.9931 & 5.9931 \\
\hline 501.190 & 5.9958 & 5.9958 & 5.9958 & 5.9958 & 5.9958 & 5.9958 \\
\hline 630.960 & 5.9973 & 5.9973 & 5.9973 & 5.9973 & 5.9973 & 5.9973 \\
\hline 794.330 & 5.9982 & 5.9982 & 5.9982 & 5.9982 & 5.9982 & 5.9982 \\
\hline 1000.00 & 5.9988 & 5.9988 & 5.9988 & 5.9988 & 5.9988 & 5.9988 \\
\hline
\end{tabular}


Table 3

Average ionization calculated using ABAKO for the range of electron densities $10^{16}-10^{21} \mathrm{~g} \mathrm{~cm}^{-3}$ and all the temperatures considered in this work.

\begin{tabular}{|c|c|c|c|c|c|c|}
\hline \multirow[t]{2}{*}{$T_{e}(\mathrm{eV})$} & \multicolumn{6}{|c|}{$N_{e}\left(\mathrm{~cm}^{-3}\right)$} \\
\hline & $10^{16}$ & $10^{17}$ & $10^{18}$ & $10^{19}$ & $10^{20}$ & $10^{21}$ \\
\hline 1.0000 & 0.7392 & 0.3178 & 0.0541 & 0.0082 & 0.0019 & 0.0012 \\
\hline 1.2600 & 0.9725 & 0.8519 & 0.4050 & 0.0832 & 0.0189 & 0.0084 \\
\hline 1.5800 & 1.0006 & 0.9747 & 0.7953 & 0.3253 & 0.1075 & 0.0399 \\
\hline 2.0000 & 1.1267 & 1.0864 & 0.9365 & 0.5648 & 0.2864 & 0.1318 \\
\hline 2.5100 & 1.6962 & 1.6505 & 1.2384 & 0.7513 & 0.4837 & 0.2987 \\
\hline 3.1600 & 1.9653 & 1.9704 & 1.7908 & 1.1532 & 0.6921 & 0.5010 \\
\hline 3.9800 & 2.1481 & 2.2967 & 2.1038 & 1.6703 & 1.0742 & 0.7366 \\
\hline 5.0100 & 2.6786 & 2.8575 & 2.7154 & 2.1035 & 1.5616 & 1.0549 \\
\hline 6.3100 & 3.0453 & 3.2680 & 3.2377 & 2.7284 & 2.0150 & 1.4317 \\
\hline 7.9400 & 3.5223 & 3.7862 & 3.7834 & 3.3739 & 2.5025 & 1.8531 \\
\hline 10.0000 & 3.8753 & 3.9561 & 3.9532 & 3.8015 & 3.0827 & 2.3342 \\
\hline 12.5900 & 3.9694 & 3.9891 & 3.9864 & 3.9277 & 3.5745 & 2.8551 \\
\hline 15.8500 & 3.9910 & 3.9965 & 3.9945 & 3.9648 & 3.7969 & 3.3143 \\
\hline 19.9500 & 3.9968 & 3.9986 & 3.9972 & 3.9795 & 3.8636 & 3.5692 \\
\hline 25.1200 & 3.9987 & 3.9994 & 3.9984 & 3.9870 & 3.9116 & 3.7492 \\
\hline 31.6200 & 4.0001 & 4.0007 & 4.0004 & 3.9934 & 3.9433 & 3.8496 \\
\hline 39.8100 & 4.0123 & 4.0177 & 4.0218 & 4.0261 & 3.9934 & 3.9366 \\
\hline 50.1100 & 4.1232 & 4.1605 & 4.1862 & 4.2362 & 4.2807 & 4.3099 \\
\hline 63.1000 & 4.5163 & 4.5824 & 4.6207 & 4.6955 & 4.7809 & 4.7971 \\
\hline 79.4300 & 4.9717 & 5.0010 & 5.0257 & 5.1001 & 5.2338 & 5.2567 \\
\hline 100.000 & 5.3914 & 5.4006 & 5.4240 & 5.5094 & 5.6499 & 5.6598 \\
\hline 125.890 & 5.7171 & 5.7210 & 5.7365 & 5.7899 & 5.8617 & 5.8473 \\
\hline 158.490 & 5.8871 & 5.8892 & 5.8967 & 5.9187 & 5.9451 & 5.9272 \\
\hline 199.530 & 5.9512 & 5.9522 & 5.9556 & 5.9648 & 5.9744 & 5.9587 \\
\hline 251.190 & 5.9768 & 5.9773 & 5.9789 & 5.9830 & 5.9866 & 5.9682 \\
\hline 316.230 & 5.9879 & 5.9882 & 5.9890 & 5.9910 & 5.9922 & 5.9788 \\
\hline 398.110 & 5.9931 & 5.9933 & 5.9938 & 5.9948 & 5.9952 & 5.9815 \\
\hline 501.190 & 5.9959 & 5.9960 & 5.9962 & 5.9968 & 5.9969 & 5.9871 \\
\hline 630.960 & 5.9974 & 5.9974 & 5.9976 & 5.9980 & 5.9979 & 5.9909 \\
\hline 794.330 & 5.9983 & 5.9983 & 5.9984 & 5.9986 & 5.9986 & 5.9936 \\
\hline 1000.00 & 5.9988 & 5.9988 & 5.9989 & 5.9990 & 5.9990 & 5.9955 \\
\hline
\end{tabular}

suits Griem's criterion [53] for each ion of the plasma charge state distribution (CSD). In Tables 2 and 3 we have listed the average ionization calculated from $\mathrm{ABAKO} /$ RAPCAL code by solving CRSS equations for several electron densities and temperatures in the ranges considered in this work. From the table it is detected that for temperatures greater than $30 \mathrm{eV}$ the average ionization is density independent for electron densities less than or equal to $10^{15} \mathrm{~cm}^{-3}$. As it is known, when $\mathrm{CE}$ is achieved the plasma magnitudes are density independent, and, therefore we could assume CE in order to calculate the average ionization for those situations. The estimation of the degree of the ionization is needed for a variety of reasons, since, for example, the plasma thermodynamic properties depend upon ionization, and, furthermore, its knowledge permits a subsequent optimization in the computation of atomic level populations in plasma and radiative properties. A detailed analysis of the charge state distributions as a function of the plasma conditions may be consulted in the previous work [33].

\subsection{Monochromatic emissivities}

In Fig. 2 we plot the spectrally resolved emissivity for an isothermal sequence $(10 \mathrm{eV})$ and several electron densities $\left(10^{13}, 10^{15}, 10^{17}\right.$ and $\left.10^{19} \mathrm{~cm}^{-3}\right)$ calculated by means of the resolution of the CRSS and SAHA equations implemented in ABAKO/RAPCAL. The average ionization is practically the same for all the cases represented, between 3.69 and 3.80, and the ions involved in the emissivity spectra are He-, Li- and Be-like ones. We can see the influence of the theoretical model (CRSS or SAHA equations) used in the emissivity calculations, i.e. the influence of the thermodynamic regime considered in the calculations. As it is known, for a given temperature the LTE regimen is achieved when the electron densities arise and this behavior is observed in Fig. 2, obtaining that for the largest density represented we can assume that LTE regime has been achieved. This result agrees with the thermodynamic regimes map shown in Fig. 1. In Fig. 3 we show the spectrally resolved emissivity for an isodense sequence $\left(10^{19} \mathrm{~cm}^{-3}\right)$ and several electron temperatures $(5,10,50$ and $100 \mathrm{eV})$. In this case the average ionization changes noticeably (between 2.10 and 5.51) and, therefore, the ions involved and the spectrum in each situation are quite different. We can also observe that the lines are more broadened than for the situations of lower densities represented in Fig. 2 which is due to the electron collisional broadening including the line profile that depends on the electron density. As in Fig. 2, we have compared CRSS and SAHA calculations. As it is known, for a given electron density as the temperature increases the departure from the LTE regime is more noticeable and this is detected in Fig. 3. Thus, for the electron temperatures of 50 and $100 \mathrm{eV}$ the plasma is in NLTE, and this result also agrees with the thermodynamic regimes map presented. 


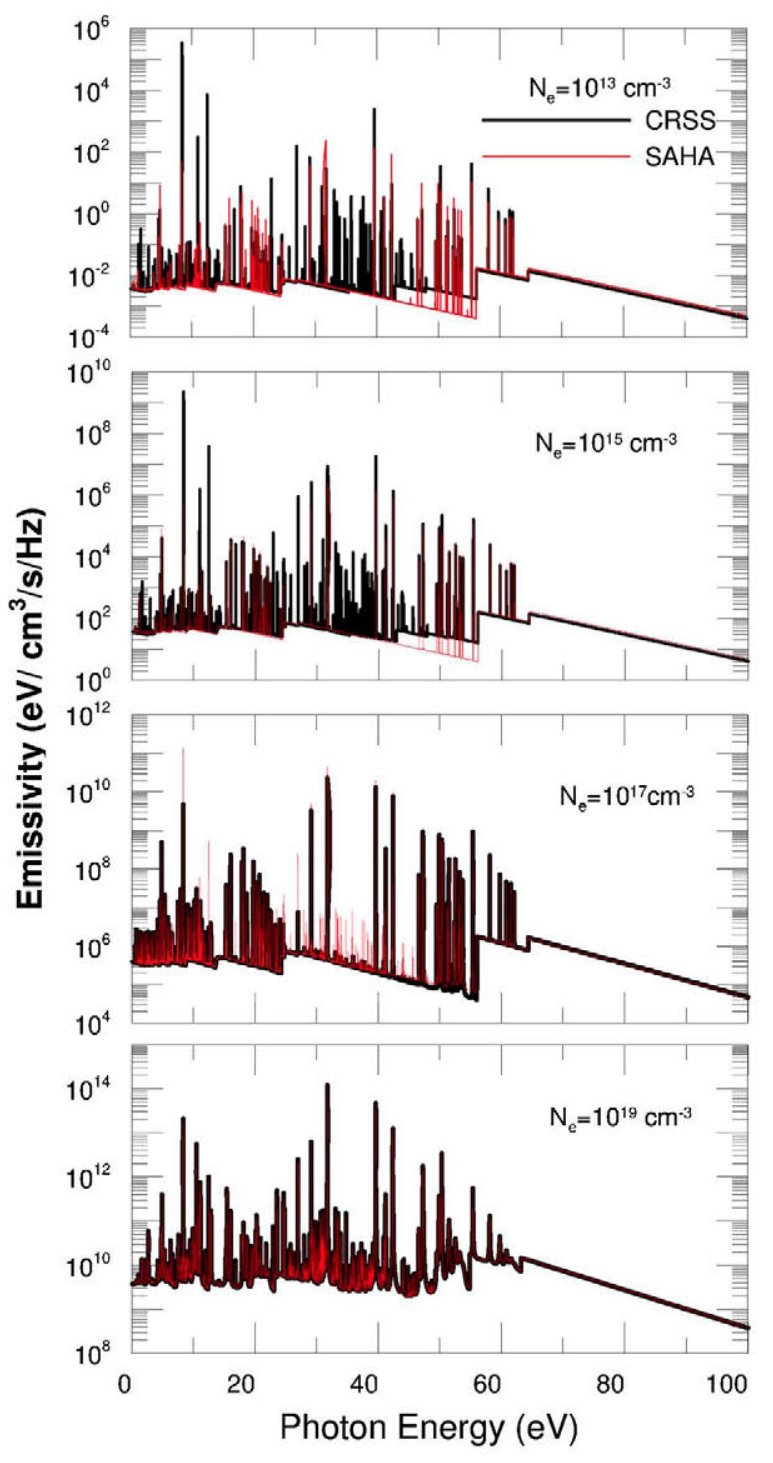

Fig. 2. Comparison between the CRSS and SAHA calculations of the monochromatic emissivities for several densities and an electron temperature of $10 \mathrm{eV}$.

\subsection{Radiative power loss and cooling rate}

Since one of the main goals of this work is to present analytical formulas for the radiative cooling rates based on polynomial fittings of the calculations of this magnitude made using ABAKO/RAPCAL, in Fig. 4 we present a comparison with results obtained using ATOMIC code [30] for the whole range of electron temperatures considered in this work and several electron densities. ATOMIC calculations are made both in configuration average (CA) and fine structure (FS) approximations, which includes intermediate-coupling and configuration-interaction effects and from the figure it is observed that at low densities and temperatures below $10 \mathrm{eV}$, the $\mathrm{CA}$ results differ considerably with respect to the FS ones. On the other hand, for temperatures higher than $10 \mathrm{eV}$ both calculations give quite similar values of the cooling rates, and, therefore, for this

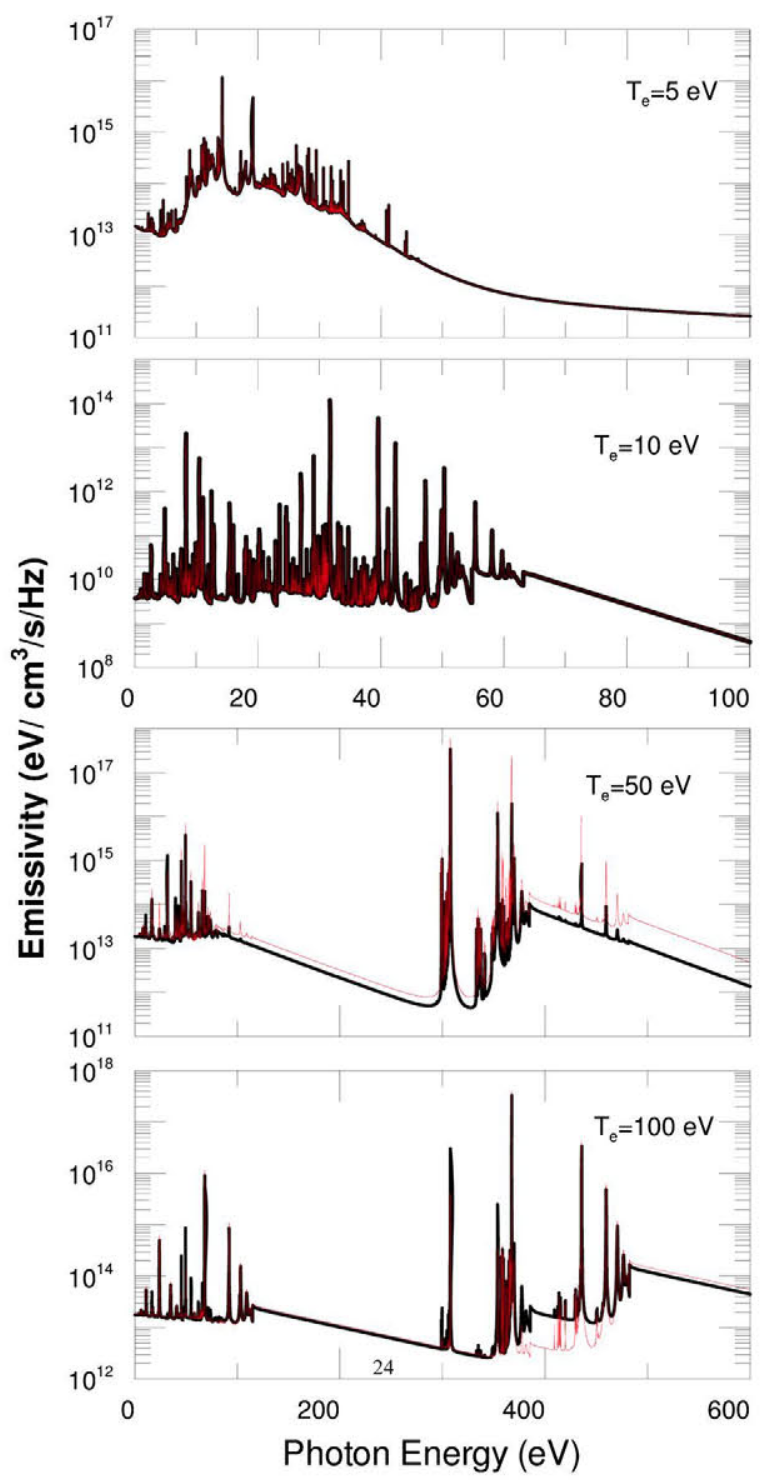

Fig. 3. Comparison between the CRSS and SAHA calculations of the monochromatic emissivities for several electron temperatures and for an electron density of $10^{19} \mathrm{~cm}^{-3}$.

range of temperatures the authors only provide the CA calculations. For this reason, although our calculations were performed in the DLA approach, we compare our results with the FS ones for temperature lower than $10 \mathrm{eV}$ and for the other temperatures with the DCA ones. In general, a good quantitative agreement is observed between them, although some discrepancies can be detected. Thus, for example, at lowest temperature $(1 \mathrm{eV})$ and for the lowest two densities $\left(10^{13}\right.$ and $\left.10^{15} \mathrm{~cm}^{-3}\right)$ we obtain differences close to a factor 2-3 between both calculations. This discrepancy could be due to the differences in the atomic data (for example in the coupling scheme considered) of the less ionized stages of carbon which are the most relevant at these plasma conditions as well as to the differences in the calculation of the rates of the processes included in both collisional-models. 

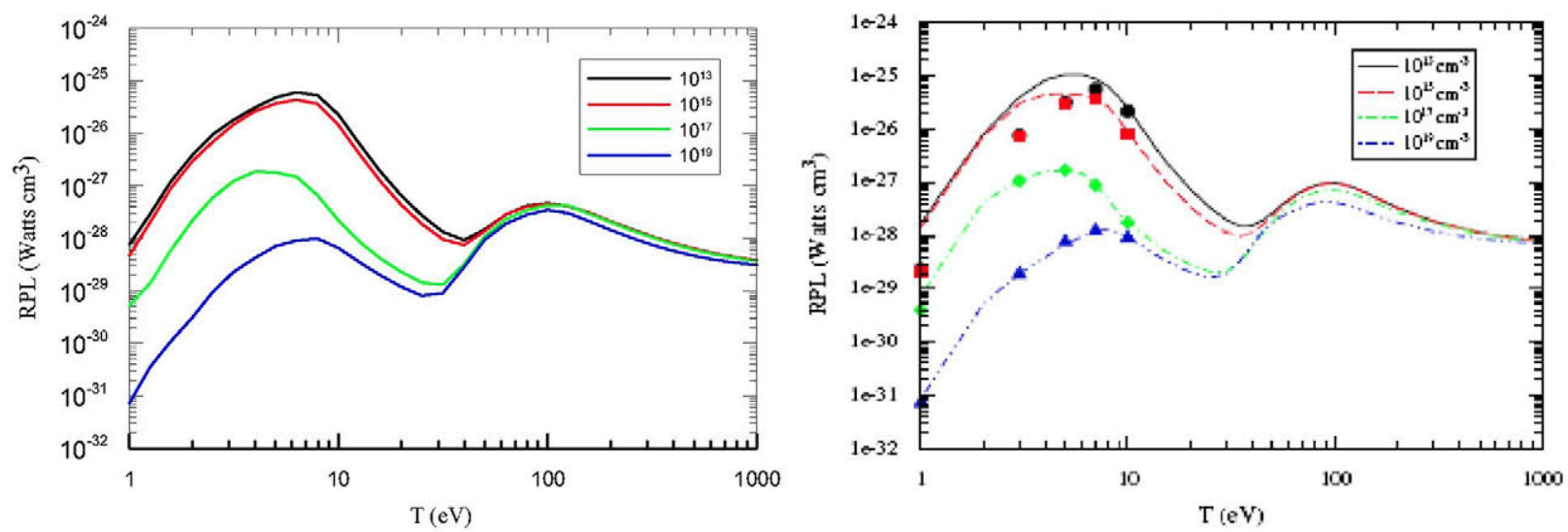

Fig. 4. Comparison between the radiative power losses normalized to obtained from ABAKO/RAPCAL (left) and ATOMIC (right) for several electron densities in $\mathrm{cm}^{-3}$.
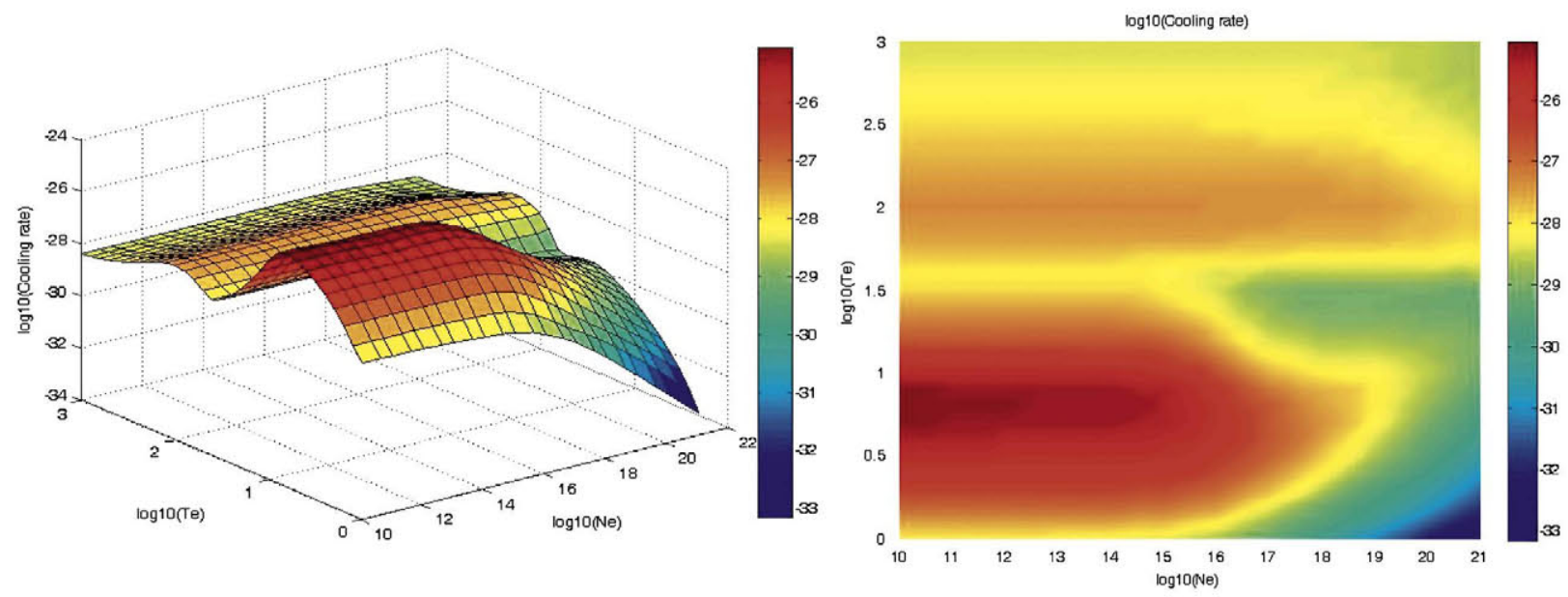

Fig. 5. Cooling rates as a function of the electron temperature and density (left) and its 2D projection (right).

In Fig. 5 and Tables 4 and 5 we present qualitative and quantitative results of the database of radiative cooling rates generated using the CRSS implemented in ABAKO/ RAPCAL employed for the subsequent polynomial fitting. From the figure a region of plasma conditions is detected in which the cooling rates are considerably lower than in other regions. This region is associated to the range of highest densities $\left(10^{18}-10^{21}\right) \mathrm{cm}^{-3}$ and temperatures up to $50 \mathrm{eV}$, as it can be seen in Table 5 . We can observe both from the figure and the tables that for temperatures greater than $200 \mathrm{eV}$ the cooling rates show a weak dependence on electron density and this behavior is more noticeable as the temperature increases. For this range of temperatures the average ionization for all the densities under analysis is very similar, around 5.9, and the most relevant ions are the $\mathrm{H}$-like and the fully stripped ones. This fact means that the free-free contribution to the cooling rates is very relevant, overall as the temperature increases, and, therefore, from Eqs. (11) and (12) it is clear, for these temperatures, their weak dependence on density. On the other hand, from the analysis of the tables of the average ionization we conclude that $\mathrm{CE}$ was a good approach for temperatures greater than $30 \mathrm{eV}$ and electron densities less than or equal to $10^{15} \mathrm{~cm}^{-3}$. This result is confirmed analyzing the values of the cooling rates in Table 4 , where we observe that for $\log T_{e}=1.50$, i.e. around $30 \mathrm{eV}$, the cooling rates are very similar in the range of electron densities. Obviously, this similarity increases with the temperature. Finally, in Fig. 5 we also detect a region of plasma conditions in which the cooling rates are relatively greater than in other regions and it corresponds to a range of low electron densities $\left(10^{10}-10^{16} \mathrm{~cm}^{-3}\right)$ and temperatures $(3-10 \mathrm{eV})$, in which the average ionization is in the interval 1.5-3.5 (see Tables 2 and 3 ) and B, Be and Li-like ions are the most abundant ones.

The influence of the thermodynamic regime on the calculation of the radiative power loss, and, therefore, in the radiative cooling rates, is analyzed in Fig. 6. In this figure we perform a comparison between the results obtained using the CRSS and the SAHA equations implemented in ABAKO/ RAPCAL for three electron densities, $\left(10^{15}, 10^{17}\right.$ and $10^{21} \mathrm{~cm}^{-3}$ ), and in the whole range of temperatures considered. For the two highest densities, LTE regime could be assumed for temperatures less than or equal to 3 and $30 \mathrm{eV}$, respectively, whereas for the lowest density represented this 
Table 4

Logarithmic of the cooling rates (in $\mathrm{W} \mathrm{cm}^{3}$ ) calculated using $\mathrm{ABAKO} /$ RAPCAL for the range of electron densities $10^{10}-10^{15} \mathrm{~g} \mathrm{~cm}^{-3}$ and all the temperatures considered in this work.

\begin{tabular}{|c|c|c|c|c|c|c|}
\hline \multirow[t]{2}{*}{$\log T_{e}(\mathrm{eV})$} & \multicolumn{6}{|c|}{$\log N_{e}\left(\mathrm{~cm}^{-3}\right)$} \\
\hline & 10 & 11 & 12 & 13 & 14 & 15 \\
\hline 0.00 & -28.03 & -28.04 & -28.05 & -28.12 & -28.21 & -28.34 \\
\hline 0.10 & -27.52 & -27.53 & -27.51 & -27.54 & -27.58 & -27.70 \\
\hline 0.20 & -26.91 & -26.91 & -26.92 & -26.93 & -26.96 & -27.05 \\
\hline 0.30 & -26.41 & -26.41 & -26.41 & -26.42 & -26.44 & -26.54 \\
\hline 0.40 & -26.00 & -26.00 & -26.01 & -26.03 & -26.06 & -26.16 \\
\hline 0.50 & -25.67 & -25.68 & -25.70 & -25.75 & -25.76 & -25.83 \\
\hline 0.60 & -25.41 & -25.42 & -25.47 & -25.52 & -25.49 & -25.59 \\
\hline 0.70 & -25.19 & -25.21 & -25.27 & -25.32 & -25.29 & -25.43 \\
\hline 0.80 & -25.07 & -25.09 & -25.17 & -25.22 & -25.21 & -25.36 \\
\hline 0.90 & -25.17 & -25.21 & -25.26 & -25.28 & -25.28 & -25.44 \\
\hline 1.00 & -25.62 & -25.64 & -25.65 & -25.65 & -25.67 & -25.85 \\
\hline 1.10 & -26.19 & -26.20 & -26.20 & -26.20 & -26.22 & -26.39 \\
\hline 1.20 & -26.73 & -26.73 & -26.73 & -26.73 & -26.75 & -26.92 \\
\hline 1.30 & -27.18 & -27.18 & -27.18 & -27.18 & -27.20 & -27.36 \\
\hline 1.40 & -27.56 & -27.56 & -27.56 & -27.56 & -27.58 & -27.73 \\
\hline 1.50 & -27.88 & -27.88 & -27.88 & -27.88 & -27.90 & -28.02 \\
\hline 1.60 & -28.04 & -28.04 & -28.04 & -28.04 & -28.05 & -28.13 \\
\hline 1.70 & -27.83 & -27.83 & -27.83 & -27.83 & -27.84 & -27.86 \\
\hline 1.80 & -27.55 & -27.55 & -27.55 & -27.55 & -27.56 & -27.55 \\
\hline 1.90 & -27.38 & -27.38 & -27.38 & -27.38 & -27.38 & -27.39 \\
\hline 2.00 & -27.33 & -27.33 & -27.33 & -27.33 & -27.33 & -27.34 \\
\hline 2.10 & -27.39 & -27.39 & -27.39 & -27.39 & -27.39 & -27.39 \\
\hline 2.20 & -27.52 & -27.52 & -27.52 & -27.52 & -27.52 & -27.52 \\
\hline 2.30 & -27.68 & -27.68 & -27.68 & -27.68 & -27.68 & -27.68 \\
\hline 2.40 & -27.83 & -27.83 & -27.83 & -27.83 & -27.83 & -27.83 \\
\hline 2.50 & -27.97 & -27.97 & -27.97 & -27.97 & -27.97 & -27.97 \\
\hline 2.60 & -28.09 & -28.09 & -28.09 & -28.09 & -28.09 & -28.10 \\
\hline 2.70 & -28.20 & -28.20 & -28.20 & -28.20 & -28.20 & -28.20 \\
\hline 2.80 & -28.29 & -28.29 & -28.29 & -28.29 & -28.29 & -28.29 \\
\hline 2.90 & -28.37 & -28.37 & -28.37 & -28.37 & -28.37 & -28.37 \\
\hline 3.00 & -28.42 & -28.42 & -28.42 & -28.42 & -28.42 & -28.42 \\
\hline
\end{tabular}

regime is not achieved at any temperature, and these results agree with the thermodynamic regimes map of Fig. 1. In previous works [32,33], we obtained that the relative deviation of both Planck and Rosseland mean radiative opacities between CRSS and SAHA calculations is less than or equal to $10 \%$ when the plasma can be assumed under LTE regime, i.e. when the criterion of Eq. (14) is fulfilled. This result is also obtained when we analyze the radiative cooling rates, as it is shown in Table 6 . However, from the table is detected that for the electron density of $10^{21} \mathrm{~cm}^{-3}$ for temperatures greater than $200 \mathrm{eV}$ the relative deviation is, in general, less than $10 \%$ which should imply that the plasma can be considered in LTE, which disagrees with the thermodynamic regimes map. This similarity is due to the fact that for this range of temperatures the main contribution to the radiative power loss is the free-free one and that in ABAKO/RAPCAL a Maxwell-Boltzmann distribution for the free electrons is considered (as many available collisional-radiative codes) but the plasma is in NLTE.

\subsection{Parametrization of the average ionization and radiative cooling rate}

Hydrodynamic simulations of plasmas require the determination of the average ionization and radiative properties for a large set of plasma conditions. However,
Table 5

Logarithmic of the cooling rates (in $\mathrm{W} \mathrm{cm}^{3}$ ) calculated using ABAKO/ RAPCAL for the range of electron densities $10^{16}-10^{21} \mathrm{~g} \mathrm{~cm}^{-3}$ and all the temperatures considered in this work.

\begin{tabular}{|c|c|c|c|c|c|c|}
\hline \multirow[t]{2}{*}{$\log T_{e}(\mathrm{eV})$} & \multicolumn{6}{|c|}{$\log N_{e}\left(\mathrm{~cm}^{-3}\right)$} \\
\hline & 16 & 17 & 18 & 19 & 20 & 21 \\
\hline 0.00 & -28.10 & -29.29 & -30.16 & -31.14 & -32.15 & -33.16 \\
\hline 0.10 & -28.10 & -28.86 & -29.58 & -30.45 & -31.42 & -32.43 \\
\hline 0.20 & -27.46 & -28.24 & -29.15 & -29.97 & -30.85 & -31.83 \\
\hline 0.30 & -26.93 & -27.67 & -28.60 & -29.52 & -30.40 & -31.33 \\
\hline 0.40 & -26.52 & -27.23 & -28.09 & -29.02 & -29.98 & -30.90 \\
\hline 0.50 & -26.21 & -26.91 & -27.76 & -28.64 & -29.56 & -30.53 \\
\hline 0.60 & -26.02 & -26.74 & -27.50 & -28.38 & -29.25 & -30.20 \\
\hline 0.70 & -25.91 & -26.75 & -27.45 & -28.15 & -29.00 & -29.91 \\
\hline 0.80 & -25.91 & -26.83 & -27.48 & -28.04 & -28.80 & -29.65 \\
\hline 0.90 & -26.12 & -27.18 & -27.69 & -28.01 & -28.66 & -29.41 \\
\hline 1.00 & -26.63 & -27.66 & -28.04 & -28.18 & -28.57 & -29.22 \\
\hline 1.10 & -27.15 & -28.07 & -28.37 & -28.45 & -28.65 & -29.11 \\
\hline 1.20 & -27.60 & -28.39 & -28.65 & -28.71 & -28.82 & -29.11 \\
\hline 1.30 & -27.97 & -28.64 & -28.88 & -28.93 & -29.01 & -29.18 \\
\hline 1.40 & -28.26 & -28.85 & -29.05 & -29.09 & -29.16 & -29.28 \\
\hline 1.50 & -28.46 & -28.89 & -29.02 & -29.04 & -29.07 & -29.18 \\
\hline 1.60 & -28.36 & -28.50 & -28.54 & -28.55 & -28.58 & -28.53 \\
\hline 1.70 & -27.92 & -27.96 & -27.99 & -28.02 & -28.07 & -28.08 \\
\hline 1.80 & -27.59 & -27.64 & -27.68 & -27.72 & -27.82 & -27.90 \\
\hline 1.90 & -27.43 & -27.46 & -27.49 & -27.55 & -27.66 & -27.76 \\
\hline 2.00 & -27.36 & -27.37 & -27.37 & -27.46 & -27.65 & -27.79 \\
\hline 2.10 & -27.38 & -27.38 & -27.41 & -27.53 & -27.76 & -27.92 \\
\hline 2.20 & -27.53 & -27.54 & -27.57 & -27.69 & -27.93 & -28.09 \\
\hline 2.30 & -27.69 & -27.70 & -27.73 & -27.85 & -28.09 & -28.25 \\
\hline 2.40 & -27.84 & -27.85 & -27.89 & -27.99 & -28.23 & -28.38 \\
\hline 2.50 & -27.98 & -27.99 & -28.02 & -28.13 & -28.35 & -28.49 \\
\hline 2.60 & -28.10 & -28.11 & -28.15 & -28.24 & -28.44 & -28.57 \\
\hline 2.70 & -28.21 & -28.22 & -28.25 & -28.34 & -28.51 & -28.62 \\
\hline 2.80 & -28.30 & -28.31 & -28.34 & -28.41 & -28.56 & -28.66 \\
\hline 2.90 & -28.37 & -28.38 & -28.40 & -28.47 & -28.59 & -28.67 \\
\hline 3.00 & -28.42 & -28.43 & -28.45 & -28.50 & -28.60 & -28.66 \\
\hline
\end{tabular}

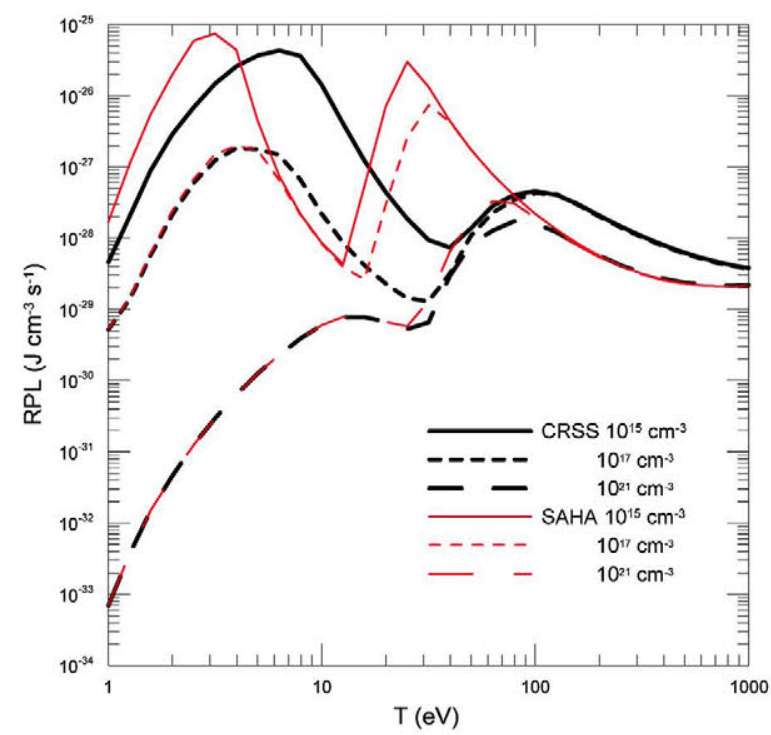

Fig. 6. Comparison between the radiative power losses obtained from ABAKO/RAPCAL using CRSS and SAHA equations for several electron densities.

their calculations involve high computational costs, and, for this reason, it is usual to make use of fitting to analytical 
Table 6

Relative deviations (in percentage) between CRSS and SAHA calculations of the radiative cooling rates for several electron densities and all the temperatures considered in this work

\begin{tabular}{|c|c|c|c|}
\hline \multirow[t]{2}{*}{$\log T_{e}(\mathrm{eV})$} & \multicolumn{3}{|c|}{$\log N_{e}\left(\mathrm{~cm}^{-3}\right)$} \\
\hline & 17 & 19 & 21 \\
\hline 0.00 & 1.18 & 0.02 & 0.01 \\
\hline 0.10 & 3.67 & 0.02 & 0.00 \\
\hline 0.20 & 6.45 & 0.00 & 0.03 \\
\hline 0.30 & 5.70 & 0.04 & 0.03 \\
\hline 0.40 & 3.66 & 0.08 & 0.02 \\
\hline 0.50 & 18.54 & 0.00 & 0.02 \\
\hline 0.60 & 9.19 & 0.12 & 0.01 \\
\hline 0.70 & 15.95 & 0.50 & 0.00 \\
\hline 0.80 & 62.83 & 2.73 & 0.06 \\
\hline 0.90 & 69.03 & 1.96 & 0.00 \\
\hline 1.00 & 61.43 & 0.47 & 0.00 \\
\hline 1.10 & 54.08 & 0.91 & 0.00 \\
\hline 1.20 & 36.18 & 0.64 & 0.00 \\
\hline 1.30 & $>100$ & 23.82 & 0.27 \\
\hline 1.40 & $>100$ & $>100$ & 9.26 \\
\hline 1.50 & $>100$ & $>100$ & $>100$ \\
\hline 1.60 & $>100$ & $>100$ & $>100$ \\
\hline 1.70 & $>100$ & $>100$ & $>100$ \\
\hline 1.80 & $>100$ & $>100$ & $>100$ \\
\hline 1.90 & 1.82 & 19.22 & 60.11 \\
\hline 2.00 & 54.11 & 41.04 & 13.44 \\
\hline 2.10 & 15.73 & 56.53 & 59.87 \\
\hline 2.20 & 71.53 & 59.94 & 3.91 \\
\hline 2.30 & 72.01 & 60.63 & 5.20 \\
\hline 2.40 & 71.19 & 59.85 & 5.53 \\
\hline 2.50 & 69.33 & 57.93 & 5.45 \\
\hline 2.60 & 66.33 & 54.75 & 5.04 \\
\hline 2.70 & 61.98 & 50.07 & 4.44 \\
\hline 2.80 & 56.35 & 44.72 & 3.73 \\
\hline 2.90 & 49.52 & 38.31 & 2.10 \\
\hline 3.00 & 41.94 & 31.59 & 2.36 \\
\hline
\end{tabular}

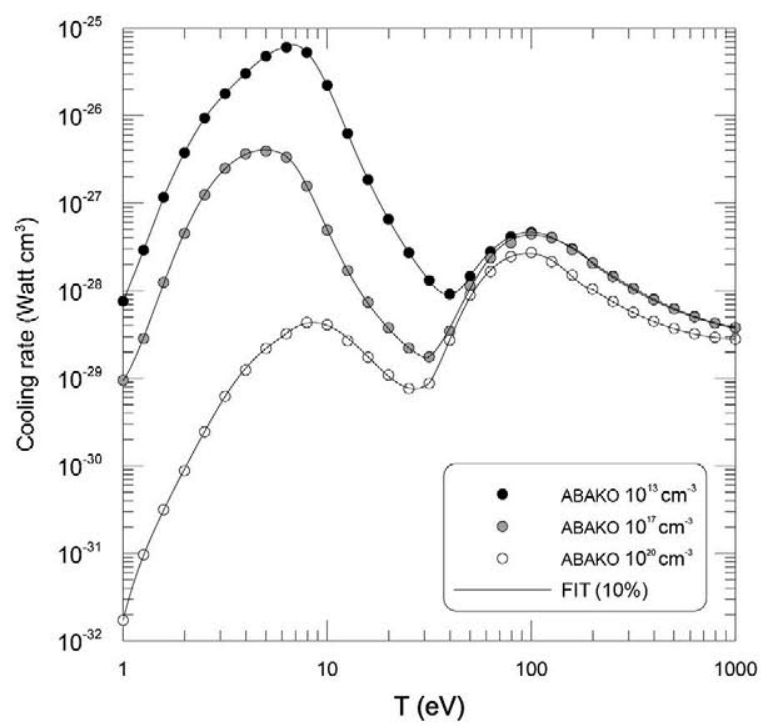

Fig. 7. Polynomial fitting of the cooling rates obtained for a relative deviation of $10 \%$

expressions of databases of those plasma quantities. In other papers [26-28] fittings of the cooling rates of carbon plasmas have been made but they are only valid for low electron density since they were obtained assuming $\mathrm{CE}$, and therefore, the fittings are density independent. However, CE regime is achieved at low electron density and high temperature and they are not accurate at larger densities or lower temperatures. In this work we present analytical expressions in powers of densities and temperatures for the calculation of the average ionization and radiative cooling rate which are valid for low to high electron densities and temperatures. The databases were generated using the CRSS model implemented in ABAKO/RAPCAL in the DLA approach.

If $A$ denotes the magnitude to fit, the analytical expression employed for the fitting is the bi-dimensional polynomial function given by Eq. (13). Taking into account that the range of electron densities and temperatures is too wide it is impossible to find only one polynomial function to make the fit of the whole range of plasma conditions, and this one must be divided into subsets to obtain a polynomial fitting in each subset. Obviously, as the error imposed becomes more restrictive the number of subsets and, therefore, of polynomial functions needed to make the fit of the whole range of plasma conditions increases. In this work we have imposed relative errors of $1 \%$ and $10 \%$ for the fittings of the average ionization and the radiative cooling rates, respectively, which gave to 28 polynomial functions to fit the average ionization and 13 for the radiative cooling rates. In order to optimize the search of the subsets of plasmas conditions and the corresponding polynomial functions a quad-tree algorithm was used. Furthermore, the maximum degrees of the polynomial was fixed to 7 , both in electron temperature and density, in order to avoid oscillating behaviors. The corresponding tables including the coefficients of the fittings are listed in Tables 7 and 8 in Appendix A where the degrees of the polynomial with respect to the temperature and electron density are $m$ and $n$ respectively (see Eq. (13)). Finally, in Fig. 7 we compare the radiative cooling rates for the whole range of electron temperatures and for two electron densities $\left(10^{13}\right.$ and $\left.10^{19} \mathrm{~cm}^{-3}\right)$ calculated from $A B A K O / R A P C A L$ code and their polynomial fittings obtained assuming relative deviations of $10 \%$.

\section{Conclusions}

In this work we performed an analysis of the influence of the thermodynamic regime in the simulation of some relevant plasma properties such as the monochromatic emissivity, the radiative power loss and the radiative cooling rates in a wide range of electron densities and temperatures. For the analysis, we first presented a map obtained using a criterion to establish the thermodynamic regime of the plasma as a whole as a function of the electron density and temperature. The results obtained with this criterion agree with those obtained using Griem's criteria for the ions and level populations. Tables of the average ionization are also presented that provide valuable information about the ions involved in each plasma condition. The influence of the thermodynamic regime on the monochromatic emissivity, the radiative power loss and the radiative cooling rates has been also analyzed. The conclusions from this analysis agree with those obtained from the 
Table 7

Parameters of the polynomial fitting for the average ionization of carbon. The degrees of the polynomial respect to the temperature and electron density are $m$ (column) and $n$ (row) respectively.

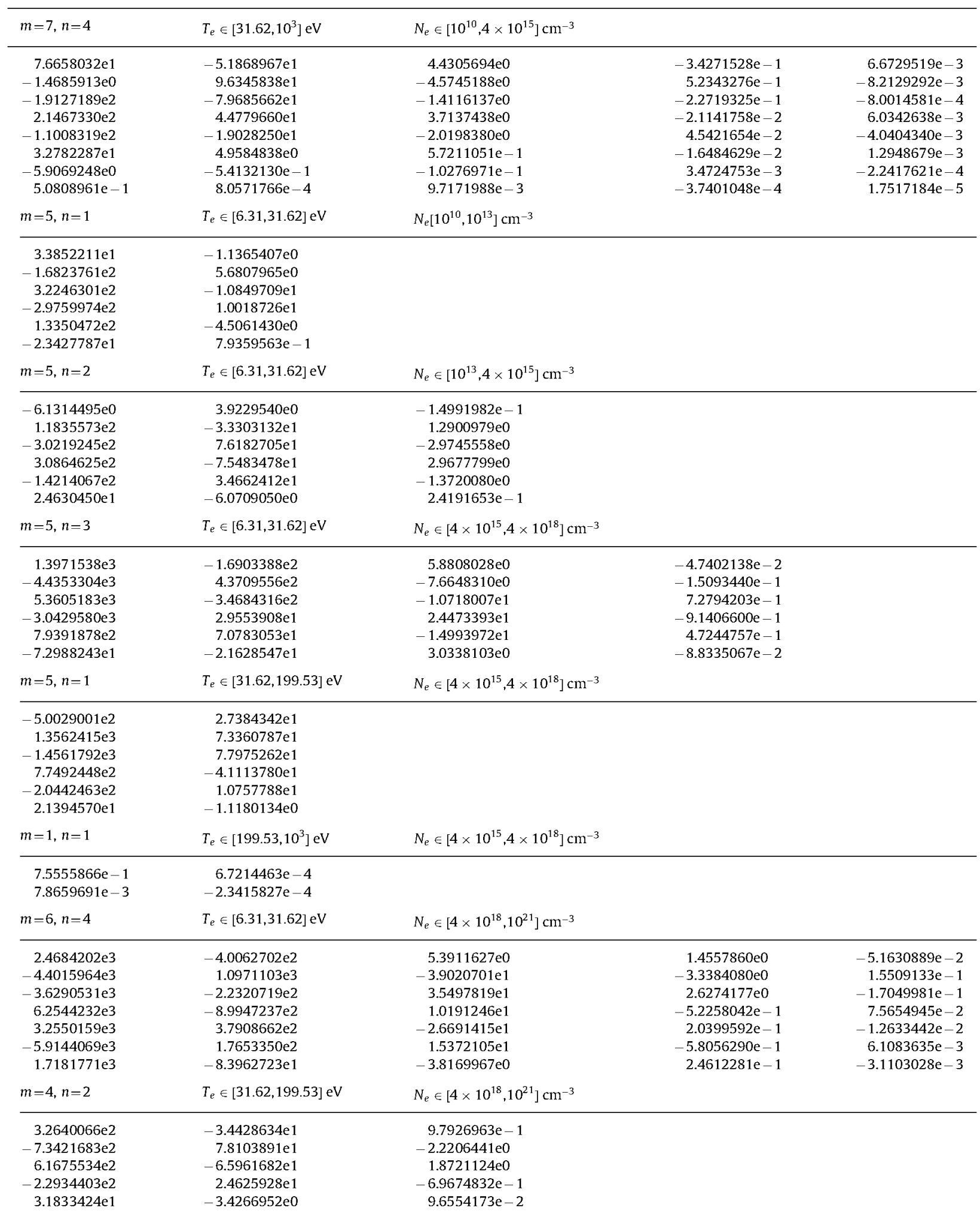


Table 7 (continued)

\begin{tabular}{|c|c|c|c|}
\hline$m=1, n=1$ & $T_{e} \in\left[199.53,10^{3}\right] \mathrm{eV}$ & $N_{e} \in\left[4 \times 10^{18}, 10^{21}\right] \mathrm{cm}^{-3}$ & \\
\hline $\begin{array}{l}7.7975801 \mathrm{e}-1 \\
5.7246665 \mathrm{e}-4\end{array}$ & $\begin{array}{r}-5.1106715 \mathrm{e}-4 \\
1.1844395 \mathrm{e}-4\end{array}$ & & \\
\hline$m=4, n=1$ & $T_{e} \in[1,2.51] \mathrm{eV}$ & $N_{\mathrm{e}} \in\left[10^{10}, 4 \times 10^{11}\right] \mathrm{cm}^{-3}$ & \\
\hline $\begin{array}{r}-2.5208768 \mathrm{e}-1 \\
3.4172960 \mathrm{e}+0 \\
-1.8914900 \mathrm{e}+1 \\
4.6108302 \mathrm{e}+1 \\
-3.9682778 \mathrm{e}+1\end{array}$ & $\begin{array}{r}7.6728730 \mathrm{e}-3 \\
-7.0796618 \mathrm{e}-2 \\
3.0549477 \mathrm{e}-1 \\
-7.1020792 \mathrm{e}-1 \\
7.4008970 \mathrm{e}-1\end{array}$ & & \\
\hline$m=4, n=1$ & $T_{e} \in[2.51,6.31] \mathrm{eV}$ & $N_{e} \in\left[10^{10}, 4 \times 10^{11}\right] \mathrm{cm}^{-3}$ & \\
\hline $\begin{array}{r}4.7258131 \mathrm{e}+0 \\
-3.4433472 \mathrm{e}+1 \\
8.9904568 \mathrm{e}+1 \\
-9.8820766 \mathrm{e}+1 \\
3.9485964 \mathrm{e}+1\end{array}$ & $\begin{array}{r}-7.0155136 \mathrm{e}-2 \\
2.9247642 \mathrm{e}-1 \\
-1.7431467 \mathrm{e}-1 \\
-4.4788783 \mathrm{e}-1 \\
4.4750371 \mathrm{e}-1\end{array}$ & & \\
\hline$m=4, n=1$ & $T_{e} \in[1.00,2.51] \mathrm{eV}$ & $N_{e} \in\left[4 \times 10^{11}, 10^{13}\right] \mathrm{cm}^{-3}$ & \\
\hline $\begin{array}{r}-5.8479081 \mathrm{e}-1 \\
8.6072701 \mathrm{e}+0 \\
-5.1912823 \mathrm{e}+1 \\
1.4215554 \mathrm{e}+2 \\
-1.4710993 \mathrm{e}+2\end{array}$ & $\begin{array}{r}3.6300712 \mathrm{e}-2 \\
-5.1537017 \mathrm{e}-1 \\
3.1265719 \mathrm{e}+0 \\
-8.9157397 \mathrm{e}+0 \\
9.9165913 \mathrm{e}+0\end{array}$ & & \\
\hline$m=4, n=1$ & $T_{e} \in[2.51,6.31] \mathrm{eV}$ & $N_{e} \in\left[4 \times 10^{11}, 10^{13}\right] \mathrm{cm}^{-3}$ & \\
\hline $\begin{array}{r}2.3714753 \mathrm{e}+1 \\
-1.6229918 \mathrm{e}+2 \\
4.0065262 \mathrm{e}+2 \\
-4.2623892 \mathrm{e}+2 \\
1.6653660 \mathrm{e}+2\end{array}$ & $\begin{array}{r}-1.6930999 \mathrm{e}+0 \\
1.1213763 \mathrm{e}+1 \\
-2.6696438 \mathrm{e}+1 \\
2.7475615 \mathrm{e}+1 \\
-1.0379133 \mathrm{e}+1\end{array}$ & & \\
\hline$m=3, n=2$ & $T_{e} \in[1.00,2.51] \mathrm{eV}$ & $N_{\mathrm{e}} \in\left[10^{13}, 4 \times 10^{14}\right] \mathrm{cm}^{-3}$ & \\
\hline $\begin{array}{r}-3.7119210 \mathrm{e}+0 \\
4.8873938 \mathrm{e}+1 \\
-2.0686276 \mathrm{e}+2 \\
3.0089768 \mathrm{e}+2\end{array}$ & $\begin{array}{r}4.9773417 \mathrm{e}-1 \\
-6.6217125 \mathrm{e}+0 \\
2.8692680 \mathrm{e}+1 \\
-4.3329138 \mathrm{e}+1\end{array}$ & $\begin{array}{r}-1.6976163 \mathrm{e}-2 \\
2.2972558 \mathrm{e}-1 \\
-1.0279175 \mathrm{e}+0 \\
1.6209812 \mathrm{e}+0\end{array}$ & \\
\hline$m=4, n=2$ & $T_{e} \in[2.51,6.31] \mathrm{eV}$ & $N_{e} \in\left[10^{13}, 4 \times 10^{14}\right] \mathrm{cm}^{-3}$ & \\
\hline $\begin{array}{r}1.5820777 \mathrm{e}+2 \\
-9.3026793 \mathrm{e}+2 \\
1.9749279 \mathrm{e}+3 \\
-1.8118240 \mathrm{e}+3 \\
6.1685036 \mathrm{e}+2\end{array}$ & $\begin{array}{r}-1.9838154 \mathrm{e}+1 \\
1.1201690 \mathrm{e}+2 \\
-2.2641856 \mathrm{e}+2 \\
1.9574624 \mathrm{e}+2 \\
-6.2205886 \mathrm{e}+1\end{array}$ & $\begin{array}{r}5.9889801 \mathrm{e}-1 \\
-3.2023979 \mathrm{e}+0 \\
6.0288436 \mathrm{e}+0 \\
-4.7240104 \mathrm{e}+0 \\
1.3135224 \mathrm{e}+0\end{array}$ & \\
\hline$m=3, n=2$ & $T_{e} \in[1.00,2.51] \mathrm{eV}$ & $N_{e} \in\left[4 \times 10^{14}, 4 \times 10^{15}\right] \mathrm{cm}^{-3}$ & \\
\hline $\begin{array}{r}-6.6623075 \mathrm{e}+0 \\
7.3207830 \mathrm{e}+1 \\
-2.0161075 \mathrm{e}+2 \\
1.0716386 \mathrm{e}+2\end{array}$ & $\begin{array}{r}8.9816751 \mathrm{e}-1 \\
-9.8517460 \mathrm{e}+0 \\
2.7178316 \mathrm{e}+1 \\
-1.5184511 \mathrm{e}+1\end{array}$ & $\begin{array}{r}-3.0562268 \mathrm{e}-2 \\
3.3680362 \mathrm{e}-1 \\
-9.4880738 \mathrm{e}-1 \\
6.0205247 \mathrm{e}-1\end{array}$ & \\
\hline$m=4, n=2$ & $T_{e} \in[2.51,6.31] \mathrm{eV}$ & $N_{e} \in\left[4 \times 10^{14}, 4 \times 10^{15}\right] \mathrm{cm}^{-3}$ & \\
\hline $\begin{array}{r}-9.2929868 \mathrm{e}+2 \\
6.8943613 \mathrm{e}+3 \\
-1.8958501 \mathrm{e}+4 \\
2.2818283 \mathrm{e}+4 \\
-1.0101306 \mathrm{e}+4\end{array}$ & $\begin{array}{r}1.2689916 \mathrm{e}+2 \\
-9.4471936 \mathrm{e}+2 \\
2.6041925 \mathrm{e}+3 \\
-3.1391073 \mathrm{e}+3 \\
1.3907512 \mathrm{e}+3\end{array}$ & $\begin{array}{r}-4.3510893 \mathrm{e}+0 \\
3.2478450 \mathrm{e}+1 \\
-8.9668118 \mathrm{e}+1 \\
1.0817131 \mathrm{e}+2 \\
-4.7933682 \mathrm{e}+1\end{array}$ & \\
\hline$m=4, n=3$ & $T_{e} \in[1.00,2.51] \mathrm{eV}$ & $N_{\mathrm{e}} \in\left[4 \times 10^{15}, 10^{17}\right] \mathrm{cm}^{-3}$ & \\
\hline $\begin{array}{r}1.9338287 \mathrm{e}+2 \\
1.6539254 \mathrm{e}+3 \\
-3.6994050 \mathrm{e}+4 \\
1.5744964 \mathrm{e}+5 \\
-1.9513713 \mathrm{e}+5\end{array}$ & $\begin{array}{r}-3.8410498 \mathrm{e}+1 \\
-2.7066895 \mathrm{e}+2 \\
6.6747450 \mathrm{e}+3 \\
-2.8812491 \mathrm{e}+4 \\
3.5898127 \mathrm{e}+4\end{array}$ & $\begin{array}{r}2.5434390 \mathrm{e}+0 \\
1.4294551 \mathrm{e}+1 \\
-3.9972255 \mathrm{e}+2 \\
1.7531770 \mathrm{e}+3 \\
-2.1970047 \mathrm{e}+3\end{array}$ & $\begin{array}{r}-5.6168541 \mathrm{e}-2 \\
-2.3934230 \mathrm{e}-1 \\
7.9377091 \mathrm{e}+0 \\
-3.5456222 \mathrm{e}+1 \\
4.4722109 \mathrm{e}+1\end{array}$ \\
\hline
\end{tabular}


Table 7 (contimued)

\begin{tabular}{|c|c|c|c|}
\hline$m=4, n=2$ & $T_{e} \in[2.51,6.31] \mathrm{eV}$ & $N_{e} \in\left[4 \times 10^{15}, 10^{17}\right] \mathrm{cm}^{-3}$ & \\
\hline $3.3142164 \mathrm{e}+1$ & $-7.9635756 \mathrm{e}+0$ & $3.3866306 \mathrm{e}-1$ & \\
\hline$-9.5954900 \mathrm{e}+2$ & $1.4496224 \mathrm{e}+2$ & $-5.0965232 \mathrm{e}+0$ & \\
\hline $4.2613868 \mathrm{e}+3$ & $-5.9160446 \mathrm{e}+2$ & $1.9767218 \mathrm{e}+1$ & \\
\hline$-6.7513522 \mathrm{e}+3$ & $9.0330152 \mathrm{e}+2$ & $-2.9440387 e+1$ & \\
\hline $3.5699239 \mathrm{e}+3$ & $-4.6786691 \mathrm{e}+2$ & $1.5026675 \mathrm{e}+1$ & \\
\hline$m=4, n=3$ & $T_{e} \in[1.00,2.51] \mathrm{eV}$ & $N_{e} \in\left[10^{17}, 4 \times 10^{18}\right] \mathrm{cm}^{-3}$ & \\
\hline$-3.5687652 \mathrm{e}+2$ & $6.0630436 \mathrm{e}+1$ & $-3.3967885 \mathrm{e}+0$ & $6.2555082 \mathrm{e}-2$ \\
\hline$-3.2360812 e+2$ & $-1.9541882 \mathrm{e}+1$ & $5.2176523 e+0$ & $-1.7184112 \mathrm{e}-1$ \\
\hline $4.3549521 \mathrm{e}+4$ & $-6.8094350 e+3$ & $3.5120842 \mathrm{e}+2$ & $-5.9707062 \mathrm{e}+0$ \\
\hline$-1.7169150 \mathrm{e}+5$ & $2.7604993 e+4$ & $-1.4695042 \mathrm{e}+3$ & $2.5891373 e+1$ \\
\hline $1.7121824 \mathrm{e}+5$ & $-2.7721107 e+4$ & $1.4865616 \mathrm{e}+3$ & $-2.6391564 \mathrm{e}+1$ \\
\hline$m=4, n=2$ & $T_{e} \in[2.51,6.31] \mathrm{eV}$ & $N_{e} \in\left[10^{17}, 4 \times 10^{18}\right] \mathrm{cm}^{-3}$ & \\
\hline $2.6423475 \mathrm{e}+3$ & $-2.9725915 e+2$ & $8.3281841 \mathrm{e}+0$ & \\
\hline$-1.8694149 \mathrm{e}+4$ & $2.1070571 \mathrm{e}+3$ & $-5.9151918 \mathrm{e}+1$ & \\
\hline $4.8210534 \mathrm{e}+4$ & $-5.4408240 \mathrm{e}+3$ & $1.5295047 \mathrm{e}+2$ & \\
\hline$-5.4071419 \mathrm{e}+4$ & $6.1087034 \mathrm{e}+3$ & $-1.7191170 \mathrm{e}+2$ & \\
\hline $2.2298440 \mathrm{e}+4$ & $-2.5214409 \mathrm{e}+3$ & $7.1023790 \mathrm{e}+1$ & \\
\hline$m=4, n=3$ & $T_{e} \in[1.00,2.51] \mathrm{eV}$ & $N_{e} \in\left[4 \times 10^{18}, 10^{20}\right] \mathrm{cm}^{-3}$ & \\
\hline $1.8627965 \mathrm{e}+1$ & $2.3456527 \mathrm{e}-1$ & $-1.5756623 \mathrm{e}-1$ & $4.6232736 \mathrm{e}-3$ \\
\hline $1.1782167 e+3$ & $-1.7580842 \mathrm{e}+2$ & $8.9017429 \mathrm{e}+0$ & $-1.5160371 \mathrm{e}-1$ \\
\hline $1.0361140 \mathrm{e}+3$ & $-3.6473930 \mathrm{e}+2$ & $2.8245373 \mathrm{e}+1$ & $-6.2888068 \mathrm{e}-1$ \\
\hline$-3.8959107 e+5$ & $6.1633191 \mathrm{e}+4$ & $-3.2455455 \mathrm{e}+3$ & $5.6881367 e+1$ \\
\hline $8.0400670 \mathrm{e}+5$ & $-1.2643731 \mathrm{e}+5$ & $6.6230111 \mathrm{e}+3$ & $-1.1554280 \mathrm{e}+2$ \\
\hline$m=4, n=2$ & $T_{e} \in[1.00,2.51] \mathrm{eV}$ & $N_{e} \in\left[10^{20}, 10^{21}\right] \mathrm{cm}^{-3}$ & \\
\hline $1.4049173 \mathrm{e}+2$ & $-1.3790245 \mathrm{e}+1$ & $3.3147983 \mathrm{e}-1$ & \\
\hline$-1.7328432 \mathrm{e}+2$ & $1.8828869 \mathrm{e}+1$ & $-4.8337427 e-1$ & \\
\hline$-2.8766051 e+3$ & $2.9275998 \mathrm{e}+2$ & $-7.4261889 \mathrm{e}+0$ & \\
\hline $9.9556665 \mathrm{e}+3$ & $-1.0510777 \mathrm{e}+3$ & $2.7431279 \mathrm{e}+1$ & \\
\hline$-9.8236386 e+3$ & $1.0619775 \mathrm{e}+3$ & $-2.8236424 \mathrm{e}+1$ & \\
\hline$m=4, n=2$ & $T_{e} \in[2.51,6.31] \mathrm{eV}$ & $N_{e} \in\left[10^{20}, 10^{21}\right] \mathrm{cm}^{-3}$ & \\
\hline $1.9595252 \mathrm{e}+3$ & $-1.8421095 e+2$ & $4.3153320 \mathrm{e}+0$ & \\
\hline$-1.5354865 e+4$ & $1.4535359 \mathrm{e}+3$ & $-3.4324414 \mathrm{e}+1$ & \\
\hline $4.3311854 \mathrm{e}+4$ & $-4.1199047 e+3$ & $9.7804495 \mathrm{e}+1$ & \\
\hline$-5.2038774 \mathrm{e}+4$ & $4.9684679 \mathrm{e}+3$ & $-1.1841092 \mathrm{e}+2$ & \\
\hline $2.2592869 e+4$ & $-2.1633083 e+3$ & $5.1710921 \mathrm{e}+1$ & \\
\hline$m=2, n=2$ & $T_{e} \in[2.51,3.98] \mathrm{eV}$ & $N_{e} \in\left[4 \times 10^{18}, 4 \times 10^{19}\right] \mathrm{cm}^{-3}$ & \\
\hline$-1.6894190 \mathrm{e}+1$ & $6.0768905 \mathrm{e}-1$ & $1.1706292 \mathrm{e}-2$ & \\
\hline$-1.8847053 e+2$ & $2.6362847 e+1$ & $-8.5711302 \mathrm{e}-1$ & \\
\hline $2.4859649 \mathrm{e}+2$ & $-3.3225834 \mathrm{e}+1$ & $1.0565682 \mathrm{e}+0$ & \\
\hline$m=2, n=2$ & $T_{e} \in[3.98,6.31] \mathrm{eV}$ & $N_{e} \in\left[4 \times 10^{18}, 4 \times 10^{19}\right] \mathrm{cm}^{-3}$ & \\
\hline$-1.2724429 \mathrm{e}+3$ & $1.3467624 \mathrm{e}+2$ & $-3.5637689 \mathrm{e}+0$ & \\
\hline $3.4802958 \mathrm{e}+3$ & $-3.6773660 \mathrm{e}+2$ & $9.7143659 \mathrm{e}+0$ & \\
\hline$-2.3782109 \mathrm{e}+3$ & $2.5117676 \mathrm{e}+2$ & $-6.6302500 \mathrm{e}+0$ & \\
\hline$m=2, n=1$ & $T_{e} \in[2.51,3.98] \mathrm{eV}$ & $N_{e} \in\left[4 \times 10^{19}, 10^{20}\right] \mathrm{cm}^{-3}$ & \\
\hline $4.6468494 \mathrm{e}+0$ & $-2.6176070 \mathrm{e}-1$ & & \\
\hline$-5.1628975 e+1$ & $2.5806884 \mathrm{e}+0$ & & \\
\hline $7.8720007 \mathrm{e}+1$ & $-3.8486646 \mathrm{e}+0$ & & \\
\hline$m=2, n=1$ & $T_{e} \in[3.98,6.31] \mathrm{eV}$ & $N_{e} \in\left[4 \times 10^{19}, 10^{20}\right] \mathrm{cm}^{-3}$ & \\
\hline $7.0007991 \mathrm{e}+0$ & $-4.5184854 \mathrm{e}-1$ & & \\
\hline$-1.7629094 \mathrm{e}+1$ & $1.1317847 \mathrm{e}+0$ & & \\
\hline $1.5501194 \mathrm{e}+1$ & $-9.0512669 \mathrm{e}-1$ & & \\
\hline
\end{tabular}


Table 8

Parameters of the polynomial fitting for cooling rate of carbon. The degrees of the polynomial respect to the temperature and electron density are $m$ (column) and $n$ (row) respectively.

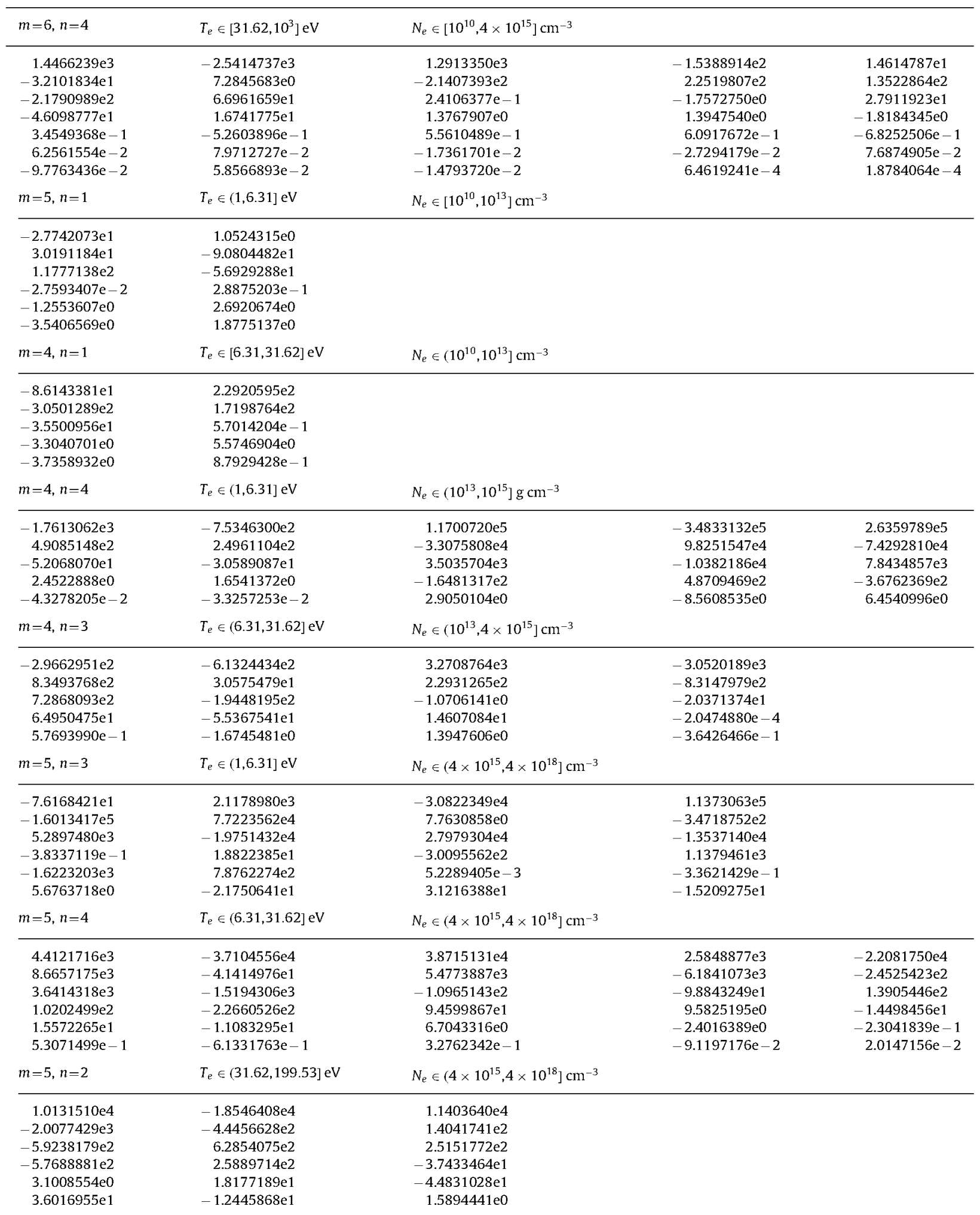


Table $\mathbf{8}$ (continued)

\begin{tabular}{|c|c|c|c|}
\hline$m=2, n=1$ & $T_{e} \in\left(199.53,10^{3}\right] \mathrm{eV}$ & $N_{e} \in\left(4 \times 10^{15}, 4 \times 10^{18}\right] \mathrm{cm}^{-3}$ & \\
\hline $\begin{array}{l}-2.1820168 \mathrm{e} 1 \\
3.2287070 \mathrm{e}-1 \\
-1.4783947 \mathrm{e}-1\end{array}$ & $\begin{array}{l}-3.0802436 \mathrm{e} 0 \\
1.4351770 \mathrm{e}-1 \\
3.1490439 \mathrm{e}-2\end{array}$ & & \\
\hline$m=5, n=2$ & $T_{e} \in(1,6.31] \mathrm{eV}$ & $N_{e} \in\left(4 \times 10^{18}, 10^{21}\right] \mathrm{cm}^{-3}$ & \\
\hline $\begin{array}{r}-1.1552539 \mathrm{e}+1 \\
-3.8894898 \mathrm{e}+3 \\
-1.0557006 \mathrm{e}+0 \\
4.2879761 \mathrm{e}+2 \\
1.2668114 \mathrm{e}-3 \\
-1.1596016 \mathrm{e}+1\end{array}$ & $\begin{array}{r}-2.7600554 \mathrm{e}+2 \\
3.4851539 \mathrm{e}+3 \\
2.8971070 \mathrm{e}+1 \\
-4.0422186 \mathrm{e}+2 \\
-7.3570790 \mathrm{e}-1 \\
1.1358117 \mathrm{e}+1\end{array}$ & $\begin{array}{r}1.7554959 \mathrm{e}+3 \\
-1.0947698 \mathrm{e}+3 \\
-1.8681069 \mathrm{e}+2 \\
1.3588710 \mathrm{e}+2 \\
4.8994054 \mathrm{e}+0 \\
-3.9978006 \mathrm{e}+0\end{array}$ & \\
\hline$m=4, n=2$ & $T_{e} \in(6.31,31.62] \mathrm{eV}$ & $N_{e} \in\left(4 \times 10^{18}, 10^{21}\right] \mathrm{cm}^{-3}$ & \\
\hline $\begin{array}{c}-4.7343766 \mathrm{e} 2 \\
4.7308890 \mathrm{e} 36 \\
-2.4029942 \mathrm{e} 2 \\
1.1034747 \mathrm{e} 2 \\
-1.0440149 \mathrm{e} 1\end{array}$ & $\begin{array}{l}3.1713026 \mathrm{e} 3 \\
-1.2186030 \mathrm{e} 3 \\
5.2642298 \mathrm{e} 2 \\
-8.8387306 \mathrm{e}-2 \\
8.8559168 \mathrm{e} 0\end{array}$ & $\begin{array}{r}-6.2768870 \mathrm{e} 3 \\
2.4338246 \mathrm{e} 1 \\
-4.1648129 \mathrm{e} 2 \\
3.9860953 \mathrm{e} 0 \\
-2.4344869 \mathrm{e} 0\end{array}$ & \\
\hline$m=6, n=3$ & $T_{e} \in(31.62,199.53] \mathrm{eV}$ & $N_{e} \in\left(4 \times 10^{18}, 10^{21}\right] \mathrm{cm}^{-3}$ & \\
\hline $\begin{array}{r}-6.7094598 \mathrm{e}+3 \\
9.5477713 \mathrm{e}+2 \\
-1.0902049 \mathrm{e}+3 \\
-7.9312370 \mathrm{e}+1 \\
4.3883969 \mathrm{e}+1 \\
-7.6747666 \mathrm{e}-1 \\
-8.6490957 \mathrm{e}-1\end{array}$ & $\begin{array}{r}1.1441974 \mathrm{e}+4 \\
1.8269760 \mathrm{e}+2 \\
6.4215382 \mathrm{e}+2 \\
1.6653356 \mathrm{e}+1 \\
-2.0726996 \mathrm{e}+1 \\
-6.7496255 \mathrm{e}-1 \\
6.3386969 \mathrm{e}-1\end{array}$ & $\begin{array}{r}-4.1065464 \mathrm{e}+3 \\
-8.9011114 \mathrm{e}+1 \\
-1.2687602 \mathrm{e}+2 \\
1.9834573 \mathrm{e}+1 \\
-6.1511503 \mathrm{e}-1 \\
8.0843216 \mathrm{e}-1 \\
-2.0540649 \mathrm{e}-1\end{array}$ & $\begin{array}{r}-1.9322320 \mathrm{e}+3 \\
5.3413978 \mathrm{e}+2 \\
1.0024735 \mathrm{e}+2 \\
-4.2687196 \mathrm{e}+1 \\
3.5249222 \mathrm{e}+0 \\
1.9866930 \mathrm{e}-1 \\
2.5736330 \mathrm{e}-2\end{array}$ \\
\hline$m=2, n=1$ & $T_{e} \in\left(199.53,10^{3}\right] \mathrm{eV}$ & $N_{e} \in\left(4 \times 10^{18}, 10^{21}\right] \mathrm{cm}^{-3}$ & \\
\hline $\begin{array}{l}-2.3720987 \mathrm{e} 1 \\
-1.4257110 \mathrm{e} 0 \\
-4.8469274 \mathrm{e}-1\end{array}$ & $\begin{array}{l}3.1804483 \mathrm{e} 0 \\
2.4400976 \mathrm{e}-1 \\
1.2570407 \mathrm{e}-1\end{array}$ & & \\
\hline
\end{tabular}

observation of the thermodynamic regimes map. Furthermore, when the criterion presented to establish the LTE regime is fulfilled the relative deviations between CRSS and SAHA calculations of the radiative cooling rates are always less than or equal to $10 \%$, as it also happened with the radiative mean opacities. Finally, parametrizations of the average ionization and the radiative cooling rates based on polynomial functions of the electron density have been also presented. These parametrizations are valid for a wide range of electron densities and temperatures.

\section{Acknowledgments}

This work has been supported by the Research Project of the Spanish Government ((ENE2009-11208/FTN), by the Research Project of the Government of the Canary Islands (SolSubC200801000057) and also by the Keep in touch Project of the European Union.

\section{Appendix A}

In this section we present the tables of the coefficients corresponding to the parametrization of the average ionization and the radiative cooling rates, Tables 7 and 8 , respectively.

\section{References}

[1] Smith HM, Verwichte E. Hot tail runaway electron generation in tokamak disruptions. Phys Plasmas 2008;15:072502.

[2] Meade DM. Effect of high-Z impurities on ignition and Lawson conditions for a thermonuclear reactor. Nucl Fusion 1974;14:289-91.

[3] Jensen RV, Post DE, Grasberger WH, Tarter CB, Lokke WA. Calculations of impurity radiation and its effects on Tokamak experiments. Nucl Fusion 1977;17:1187-96.

[4] Tokar MZ, Kelly FA. The role of plasma-wall interactions in thermal instabilities at the tokamak edge. Phys Plasmas 2003;10:4378-86.

[5] Tokar MZ. Impurity transport and radiation. Fusion Sci Technol 2008:53:243-50

[6] Lazarus EA, Bell JD, Bush CE, Carnevali A, Dunlap JL, Edmonds Ph, et al. Confinement improvement in beam heated ISX-B discharges with low-Z impurity injection. J Nucl Mater 1984;121:61-8.

[7] Ongena J, Messiaen AM, Tokar M, Samm U, Unterberg B, Dumortier $P$, et al. Results and modeling of high-power edge radiation cooling in TEXTOR. Phys Scr 1995;52:449-57.

[8] Rozhansky V, Senichenkov I, Veselova I, Morozov D, Schneider R. Penetration of supersonic gas jets into a tokamak. Nucl Fusion 2006;46: 367-82.

[9] Gal K, Feher T, Smith H, Fulop T, Helander P. Runaway electron generation during plasma shutdown by killer pellet injection. Plasma Phys Control Fusion 2008:50:055006.

[10] Mignone A. The dynamics of radiative shock waves: linear and nonlinear evolution. Astrophys J 2005;626:373-88.

[11] Busquet M, Audit E, Gonzalez M, Stehlé C, Thais F, Acef o, et al. Effect of lateral radiative losses on radiative shock propagation. High Energy Density Phys 2007;3:8-11.

[12] Falize E, Bouquet $S$, Michaut $C$. Radiation hydrodynamics scaling laws in high energy density physics and laboratory astrophysics. J Phys: Conf Ser 2008;112:042016. 
[13] Michaut C, Falize E, Cavet C, Bouquet S, Koenig M, Vinci T, et al. Classification of and recent research involving radiative shocks. Astrophys Space Sci 2009:32:77-84.

[14] Keilty KA, Liang EP, Ditmire T, Remington BA, Shigemori K, Rubenchik AM. Modeling of laser-generated radiative blast waves. Astrophys J 2000;538:645-52.

[15] Edwards MJ, MacKinnon AJ, Zweiback J, Shigemori K, Ryutov D, Rubenchik AM, et al. Investigation of ultrafast laser-driven radiative blast waves. Phys Rev Lett 2001;87:085004.

[16] Laming JM, Grun J. Dynamical overstability of radiative blast waves: the atomic physics of shock stability. Phys Rev Lett 2002;89:125002.

[17] Keiter PA, Drake RP, Perry TS, Robey HF, Remington BA, Iglesias CA, et al. Observation of a hydrodynamically driven, radiativeprecursor shock. Phys Rev Lett 2002;89:165003.

[18] Bouquet $S$, Stehle C, Koenig M, Chieze JP, Benuzzi-Mounaix A, Batani $D$, et al. Observation of laser driven supercritical radiative shock precursors. Phys Rev Lett 2004;92:225001.

[19] Fadeyev YA, Gillet D. The structure of radiative shock waves. II. The multilevel hydrogen atom. Astron Astrophys 2000;354:349-64.

[20] C. Stehle, J.P. Chieze, in: F. Combes, D. Barret (Eds.), SF2A-2002: Semaine de l'Astrophysique Francaise, Paris, France, EDP Sciences, Les Ulis; 2002. p. 493.

[21] Shigemori K, Kodama R, Farley DR, Koase T, Estabrook KG, Remington $\mathrm{BA}$, et al. Experiments on radiative collapse in laser-produced plasmas relevant to astrophysical jets. Phys Rev E 2000;62: 8838-41.

[22] Lebedev SV, Chittenden JP, Beg FN, Bland SN, Ciardi A, Ampleford D, et al. Laboratory astrophysics and collimated stellar outflows: the production of radiatively cooled hypersonic plasma jets. Astrophys 2002;564:113-9.

[23] Hicks AK, Canizares CR. Effects of departures from ionization equilibrium on cooling-flow X-ray spectra. Astrophys J 2001;556: $468-70$.

[24] Pope ECD, Pavlovski G, Kaiser CR, Fangohr H. The effects of thermal conduction on the intracluster medium of the Virgo cluster. Mon Not R Astron Soc 2005;364:13-28.

[25] Peterson JR, Fabian AC. X-ray spectroscopy of cooling clusters. Phys Rep 2006;427:1-39.

[26] Post DE, Jensen RV, Tarter CB, Grasberger WH, Lokke WA. Steadystate radiative cooling rates for low density, high temperature plasmas. Atom Data Nucl Data 1977;20:397-439.

[27] Summers HP, McWhirter RWP. Radiative power loss from laboratory and astrophysical plasmas. I. Power loss from plasmas in steady-state ionisation balance. J Phys B: Atom Mol Phys 1979;12: 2387-411.

[28] Schure KM, Kosenko D, Kaastra JS, Keppens R, Vink J. A new radiative cooling curve based on an up-to-date plasma emission code. Astron Astrophys 2009;508 751-U240.

[29] Clark R, Abdallah J, Post D. Radiation rates for low-Z impurities in edge plasmas. J Nucl Mater 1995;220-222:1028-32.

[30] Colgan J, Fontes CJ, Abdallah J. Collisional-radiative studies of carbon plasmas. High Energy Density Phys 2006;2:90-6.

[31] Fournier KB, May MJ, Pacella D, Finkenthal M, Gregory BC, Goldstein WH. Calculation of the radiative cooling coefficient for krypton in a low density plasma. Nucl Fusion 2000;40:847-64.

[32] Gil JM, Rodriguez R, Florido R, Rubiano JG, Martel P, Minguez E. Determination of corona, LTE and NLTE regimes of optically thin carbon plasmas. Laser Part Beams 2008;26:21-31.

[33] Gil JM, Rodriguez R, Martel P, Florido R, Rubiano JG, Mendoza MA, et al. Analysis of the influence of the plasma thermodynamic regime in the spectrally resolved and mean radiative opacity calculations of carbon plasmas in a wide range of density and temperature. J Quant Spectrosc Radiat Transfer 2012 〈http://dx. doi.org/10.1016/j.jqsrt.2012.05.016).

[34] Rodriguez R, Florido R, Gil JM, Rubiano JG, Martel P, Minguez E. RAPCAL code: a computational package to compute radiative properties for optically thin and thick low and high-z plasmas in a wide range of density and temperature. Laser Part Beams 2008;26:433-48.

[35] Florido R, Rodríguez R, Gil JM, Rubiano JG, Martel P, Minguez E et al. Modeling of population kinetics of plasmas that are not in local thermodynamic equilibrium, using a versatile collisionalradiative model based on analytical rates. Phys Rev E 2009;80: 056402.

[36] Rodriguez R, Florido R, Gil JM, Rubiano JG, Suarez D, Martel P, et al Collisional-radiative calculations of optically thin and thick plasmas using the computational package ABAKO/RAPCAL. Commun Comput Phys 2010;8:185-210.

[37] Bowen C, Lee RW, Ralchenko Yu. Comparing plasma population kinetics codes: review of the NLTE-3 Kinetics Workshop. J Quant Spectrosc Radiat Transfer 2006;99:102-19.

[38] Rubiano JG, Florido R, Bowen C, Lee RW, Ralchenko Y. Review of the 4 th NLTE code comparison workshop. High Energy Density Phys 2007;3:225-32.

[39] Fontes CJ, Abdallah J, Bowen C, Lee RW, Ralchenko Y. Review of the NLTE-5 kinetics workshop. High Energy Density Phys 2009;5: $15-22$.

[40] Lotz W. Electron-impact ionization cross sections and ionization coefficients for atoms and ions from hydrogen to calcium. Z Phys $1968 ; 216: 241-7$.

[41] Van Regemorter HV. Rate of collisional excitation in stellar atmospheres. Astrophys J 1962;136:906-15.

[42] Kramers HA. On the theory of X-ray absorption and of the continuous X-ray spectrum. Philos Mag 1923;46:836-71.

[43] Griem HR. Principles of plasma spectroscopy. Cambridge: Cambridge University Press; 1997. p. 177-9.

[44] Gu MF. The flexible atomic code. Can J Phys 2008;86:675-89.

[45] Rodriguez R, Florido R, Gil JM, Rubiano JG, Martel P, Mínguez E Influence of the atomic description and configuration interaction effects on collisional-radiative calculations of low ionized carbon plasmas. J Quant Spectrosc Radiat Transfer 2009;110:2191-207.

[46] Stewart JC, Pyatt KD. Lowering of ionization potentials in plasmas. Astrophys J 1966;144:1203-11.

[47] Cowan RD. The theory of atomic structure. Berkeley: University of California Press; 1981. p. 395-7.

[48] Dimitrijevic MS, Konjevic N. Simple estimates for Stark-broadening of ion lines in stellar plasmas. Astron Astrophys 1987;172:345-9.

[49] Rose SJ. Calculation of the radiative opacity of laser-produced plasma. J Phys B: At Mol Opt Phys 1992;28:1667-81.

[50] Chung HK, Fournier KB, Lee RW. Non-LTE kinetics modeling of krypton ions: calculations of radiative cooling coefficients. High Energy Density Phys 2006;2:7-15.

[51] Karzas WJ, Latter R. Electron radiative transitions in a Coulomb field. Astrophys J Suppl 1961;6:167-212.

[52] Rodriguez R, Gil JM, Espinosa G, Florido R, Rubiano JG, Mendoza $\mathrm{MA}$, et al. Determination and analysis of plasma parameters for simulations of radiative blast waves launched in clusters of xenon and krypton. Plasma Phys Control Fusion 2012;54:045012.

[53] Griem HR. Validity of local thermodynamic equilibrium in plasma spectroscopy. Phys Rev 1963;131:1170-6. 